\title{
Candidate effectors contribute to race differentiation and virulence of the lentil anthracnose pathogen Colletotrichum lentis
}

\author{
Vijai Bhadauria, Ron MacLachlan, Curtis Pozniak and Sabine Banniza*
}

\begin{abstract}
Background: The hemibiotroph Colletotrichum lentis, causative agent of anthracnose on Lens culinaris (lentil) was recently described as a new species. During its interaction with the host plant, $C$. lentis likely secretes numerous effector proteins, including toxins to alter the plant's innate immunity, thereby gaining access to the host tissues for nutrition and reproduction.

Results: In silico analysis of 2000 ESTs generated from C. lentis-infected lentil leaf tissues identified 15 candidate effectors. In planta infection stage-specific gene expression waves among candidate effectors were revealed for the appressorial penetration phase, biotrophic phase and necrotrophic phase. No sign of positive selection pressure $[\omega(\mathrm{dN} / \mathrm{dS})<1]$ in effectors was detected at the intraspecific level. A single nucleotide polymorphism in the ORF of candidate effector CICE6, used to develop a KASPar marker, differentiated perfectly between pathogenic race 0 and race 1 isolates when tested on 52 isolates arbitrarily selected from a large culture collection representing the western Canadian population of C. lentis. Furthermore, an EST encoding argininosuccinate lyase (Arg) was identified as a bacterial gene. A toxin protein CIToxB was further characterized as a potential host-specific toxin through heterologous in planta expression. The knock-down of CIToxB transcripts by RNAi resulted in reduced virulence, suggesting that CITOXB is a virulence factor. In silico analysis of the CIToxB sequence and comparative genomics revealed that ToxB is unlikely a foreign gene in the $C$. lentis genome. Incongruency between established species relationships and that established based on gene sequence data confirmed ToxB arose through evolution from a common ancestor, whereas the bacterial gene Arg identified in C. lentis was horizontally transferred from bacteria.

Conclusions: EST mining and expression profiling revealed a set of in planta expressed candidate effectors. We developed a KASPar assay using effector polymorphism to differentiate $C$. lentis races. Comparative genomics revealed a foreign gene encoding a potential virulence factor Arg, which was horizontally transferred from bacteria into the genus Colletotrichum. CIToxB is further characterized as a host-specific toxin that is likely to contribute to quantitative differences in virulence between the races 0 and 1 .
\end{abstract}

Keywords: Fungal pathogenicity and virulence, Plant disease resistance, Extended phenotype, Colletotrichum

\section{Background}

Colletotrichum truncatum (Schwein.) Andrus \& W.D. Moore was originally identified as the causal agent of anthracnose disease on many legume species, including lentil, soybean, faba bean and pea [1]. However, recent evidence suggests that isolates from several of these hosts were misclassified [2, 3], and Colletotrichum isolates from lentil

\footnotetext{
* Correspondence: sabine.banniza@usask.ca

Crop Development Centre/Department of Plant Sciences, University of Saskatchewan, Saskatoon, SK S7N 5A8, Canada
}

(Lens culinaris Medik.) were re-classified as C. lentis Damm as part of the destructivum clade [4]. This pathogen initiates infection through single-celled conidia that attach to the aerial parts of the host plants, and germinate to form appressoria instrumental in the mechanical breaching of the host surface. Thin penetration pegs arising from beneath the appressoria pierce the host cuticle and cell wall, and differentiate into large bulbous invasive primary hyphae that are biotrophic in nature. Plasmolyzed plant cells colonized by $C$. lentis show a weak interaction between the biotrophic hyphae and the plasma membrane [5]. The

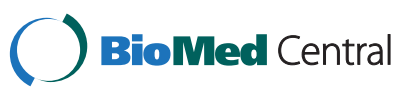

(c) 2015 Bhadauria et al. Open Access This article is distributed under the terms of the Creative Commons Attribution 4.0 International License (http://creativecommons.org/licenses/by/4.0/), which permits unrestricted use, distribution, and reproduction in any medium, provided you give appropriate credit to the original author(s) and the source, provide a link to the Creative Commons license, and indicate if changes were made. The Creative Commons Public Domain Dedication waiver (http://creativecommons.org/publicdomain/zero/1.0/) applies to the data made available in this article, unless otherwise stated. 
primary hyphae of $C$. lentis are entirely confined to the first infected epidermal cells throughout the biotrophic phase. After an initial period of biotrophic infection, the morphogenetic differentiation of thin filamentous necrotrophic secondary hyphae from the thick biotrophic primary hyphae occurs and this transition, referred to as the biotrophynecrotrophy switch, coincides with large scale cell death and tissue collapse. At this stage, anthracnose lesions become evident on the aerial parts of plants, and thousands of conidia are eventually produced in acervuli developing in these lesions, which reinitiate the disease cycle.

Two pathogenic races were described in the Canadian population of C. lentis [6]. Although not characterized by the classical hypersensitive response that limits infection of an avirulent race of a biotrophic pathogen, isolates of the less virulent race 1 of $C$. lentis showed lower conidial germination and formation of appressoria, and differences in the speed and extend of destruction during the necrotrophic phase after inoculation onto a partially resistant lentil cultivar compared to isolates of the more virulent race 0 [7].

Plant pathogenic fungi secrete small proteinaceous and non-proteinaceous molecules in their hosts to manipulate host cell structure and function, thereby facilitating infection (virulence factors and toxins) or triggering host plant defense responses (avirulence factors and elicitors) or both [8-10]. Some of these effectors exert their activity in the apoplast where they may interfere with host plant defense processes, e.g. by inhibiting plant proteases and lytic enzymes. Others are trafficked into host cells, the mechanism of which in terms of traversal of the plasma membrane has recently been disclosed. The role of the amino terminal RxLR and dEER motifs of analogous oomycete effectors in host cell entry has been established [11]. Variants of the RxLR motif have been identified in five fungal effectors (AvrL567, AvrM, Avr2, AvrLm6 and AvrPita), which bind to the phospholipid phosphatidylinositol-3-phosphate located at the exterior leaflet of the plant cell plasma membrane. This binding may facilitate the uptake of effectors through endocytosis [12]. Cytoplasmic effectors can suppress the first layer of inducible defense known as pathogen associated molecular patterns-triggered immunity (PTI) that is activated when conserved pathogen molecules, such as flagellin, EF-Tu, peptidoglycan and chitin are perceived by pathogen recognition receptors located at the cell surface. Suppression occurs by interfering with the signal transduction pathways involved in the manifestation of host defense responses. However, in resistant plants, these cytoplasmic effectors are recognized by disease resistance proteins, most of which possess nucleotide binding and leucine rich repeat domains, thus eliciting effector-triggered immunity (ETI). ETI is an accelerated and amplified PTI response, resulting in disease resistance and usually a localized hypersensitive cell death response (HR) at the infection site $[13,14]$. The role of toxins is well documented for necrotrophic pathogens, such as Stagonospora nordorum and Pyrenophora tritici-repentis. The $\tan$ spot pathogen $P$. tritici-repentis delivers two proteinaceous toxins PtrToxA and PtrToxB, and one nonproteinaceous toxin PtrToxC into wheat cells to condition virulence. PtrToxA induces necrosis in wheat genotypes carrying the toxin receptor Tsn1 whereas PtrToxB and PtrToxC elicit chlorosis in different wheat genotypes. Based on their ability to produce these toxins, eight $P$. tritici-repentis races have been described on wheat $[15,16]$.

During evolution of their genomes, plant pathogenic fungi have also acquired foreign genes through horizontal gene transfer (HGT) from both eukaryotes and prokaryotes. HGT is the nonsexual stable transmission of genetic material between genomes of different species [17, 18], and transferred genes are in most cases involved in the interaction with the host plant [19]. One such example is the transfer of PtrToxA from S. nodorum to P. triticirepentis allowing this fungus to become a major pathogen of wheat [20].

Studies conducted to date have identified a handful of candidate effectors from Colletotrichum spp. A proline rich glycoprotein, Colletotrichum intracellular hyphae 1 (CIH1) was detected in the biotrophic interface of C. lindemuthianum and bean cells [21]. The CIH1 peptide sequence contains lysin motifs, which recognize and bind to $\mathrm{N}$-acetyl D-glucosamine, and thus may play a role in protecting fungal chitin from plant chitinases, or in camouflaging the fungus from being detected by the basal defense system [22]. The role of the effector CgDN3 in suppressing HR induced by C. gloeosporioides in the tropical pasture legumes Stylosanthes guianensis was confirmed by targeted gene disruption. The $\triangle C g D N 3$ mutants elicited a localized HR in host plants, suggesting a role of $C g D N 3$ in averting HR in susceptible hosts during the biotrophic phase of fungal infection [23]. A yeast signal sequence trap cDNA library was constructed from in vitro grown mycelia of C. graminicola, the causal agent of stem rot and leaf anthracnose on maize to capture genes encoding secretory proteins, and 103 unique sequences were identified as secretory protein-encoding genes [24]. To identify secretory proteins potentially involved in the virulence of C. higginsianum on host plants like Arabidopsis and brassicas, a cDNA plasmid library was constructed using RNA isolated from in vitro formed appressoria, and fifty-three unique sequences were predicted to encode putative secretory proteins, including 26 secretory proteins lacking a transmembrane domain (extracellular secretory proteins) [25]. Takahara et al. [26] developed a fluorescenceactivated cell sorting (FACS) method to purify the intracellular biotrophic hyphae from C. higginsianum-infected 
Arabidopsis leaves, constructed a biotrophy-specific cDNA library, and identified a set of ESTs encoding putative secretory proteins. We previously constructed a cDNA library from the biotrophy-necrotrophy switch of a $C$. lentis isolate and identified 122 unique sequences encoding potential secretory proteins, including eleven candidate effectors [5]. Among them was the effector protein CtNUDIX, a potential biotrophy-necrotrophy switch regulator, that was expressed precisely before the switch from biotrophy to necrotrophy and induced HR. Overexpression of the CtNU$D I X$ in $C$. lentis and the rice blast pathogen $M$. oryzae resulted in incompatibility with their host plants, suggesting a potential role of this biotrophy-necrotrophy switch-specific effector in establishing hemibiotrophy [27]. Deep sequencing of the C. higginsianum transcriptome associated with penetrating appressoria, FACS-isolated biotrophic hyphae, and the in planta late necrotrophy, yielded 327 unique sequences encoding secreted extracellular proteins. Among these were 198 unigenes encoding Colletotrichum-specific effector candidates, of which 102 were absent in the necrotrophy-associated transcriptome, and thus were considered as biotrophy-associated candidate effectors implicated in the establishment of biotrophy (appressorium penetration and development of biotrophic hyphae) [28]. Using fluorescent protein tagging and immunogold transmission electron microscopy, the authors showed a focal secretion of effectors during penetration via appressorium, likely to establish biotrophy. In addition, antagonistic effectors (inducing or suppressing plant cell death) were identified in the study. O'Connell et al. [3] analyzed the entire genomes and transcriptomes of $C$. higginsianum and of C. graminicola, and concluded that effectors and secondary metabolism enzymes are induced before penetration and during biotrophy, whereas hydrolyzing enzymes and transporters are active during the biotrophynecrotrophy switch.

The objectives of the present study were to identify candidate effectors among 2000 expressed sequence tags (ESTs) generated from C. lentis-infected lentil leaf tissues, understand their expression pattern during pathogenesis and develop Kompetitive Allele Specific PCR (KASPar) markers to differentiate $C$. lentis races. Fifteen candidate effectors putatively secreted by $C$. lentis during the colonization of lentil were identified, and quantitative RTPCR was performed to profile their expression in an infection time-course. Correlation of expression profiles with race identity was assessed. Homologs were found for 10 of the candidate effectors in other Colletotrichum spp. that displayed high peptide sequence identity. We also characterized the toxin gene $C l T o x B$ through heterologous agroinfiltration in tobacco and RNAi, and showed that it is likely a host-specific toxin and a virulence factor. Homologs were found in five fungal species including three in Colletotrichum. While identifying at least one HGT event (ClArg encoding argininosuccinate lyase) from bacteria into the genus Colletotrichum, comparative genomics analysis revealed that $C l T o x B$ is a native gene and not horizontally transferred from other species.

\section{Results}

Identification of $C$. lentis candidate effectors

In a previous study, we constructed a directional cDNA plasmid library from Eston (no resistance to anthracnose) leaf tissues infected with $C$. lentis isolate CT-21 (race 1) undergoing the morphogenetic biotrophy-necrotrophy transition [5]. In the present study, 2000 previously uncharacterized ESTs were sequenced and subjected to BLASTX analysis, and 780 ESTs were identified as ESTs of fungal origin. The remaining ESTs either were of plant origin ( $\sim 53 \%)$ or unknown ( 4 \%). The ORF finder, and SignalP and iPSORT algorithms were employed to identify open reading frames (ORFs) containing putative signal peptide (SP) sequences. Twenty-two ORFs were predicted to encode proteins with a putative N-terminal SP for secretion. Out of these 22 ESTs, 13 ESTs represented unique sequences (unigenes) and the remaining 9 ESTs were assembled into two individual unique sequences (Table 1) and were deposited in the NCBI GenBank EST database (dbEST). The average GC content of these unigenes was close to $59 \%$.

The translated amino acid sequences of the putative secretory proteins were analyzed for features indicative of secretory proteins that are likely to enter into host cells. No transmembrane helices were detected in these proteins, and we considered them soluble secretory proteins (referred to hereafter as candidate effectors). However, Nand O-glycosylation sites were predicted in the peptide sequences of 13 candidate effectors indicating potential for their attachment to the fungal cell membranes and cell walls. The $\mathrm{N}$ - and $\mathrm{O}$-glycosylation sites allow for attachment of sugar chains to asparagine residues, and serine and threonine residues, respectively.

The BLASTP algorithm was used to identify putative functions of these candidate effectors. Of 15 candidate effectors, ten (ClCE1 through 4, ClCE6, ClCE7, ClCE10 through 12, ClCE18) were identified as hypothetical proteins (Table 1). Two candidate effectors (ClCE9 and ClCE15) had no significant BLASTP match at a cut-off of $E$ value $\leq 1 \mathrm{e}-6$. These were queried with TBLASTN against 68,986 unigenes derived from 22 fungal and oomycete species in the COGEME EST database, and finally considered as $C$. lentis orphan sequences due to lack of significant matching hits. However, both candidate effectors were perfectly mapped onto the recently assembled C. lentis isolate CT-30 (race 0) draft genome (unpublished data). The remaining three showed significant similarity to collagen-like protein Mcl1 (ClCE5), fasciclin domain-containing protein (ClCE8) and laccase-1 
Table 1 Colletotrichum lentis candidate effectors

\begin{tabular}{llllllll}
\hline Effector & GenBank ID & Peptide (aa) & N/O-Gly & Putative function & Accession & Organism & E value \\
\hline CICE1 & JK998669 & 189 & $2 / 4$ & Hypothetical protein & EFQ27227 & Glomerella graminicola & $3.00 E-53$ \\
CICE2 & JK998670 & 176 & $1 / 22$ & Hypothetical protein & EFQ26411 & Glomerella graminicola & $3.00 E-26$ \\
CICE3 & JK998671 & 153 & $2 / 5$ & Hypothetical protein & EFQ27227 & Glomerella graminicola & $7.00 E-33$ \\
CICE4 & JK998672 & 236 & $1 / 2$ & Secreted protein & EGG09255 & Melampsora larici-populina & $1.00 E-08$ \\
CICE5 & JK998673 & 167 & $1 / 1$ & Collagen-like protein Mcl1 & EFY89687 & Metarhizium acridum & $2.00 E-14$ \\
CICE6 & JK998674 & 249 & $1 / 5$ & Hypothetical protein & EFQ33016 & Glomerella graminicola & $3.00 E-148$ \\
CICE7 & JK998675 & 132 & $1 / 5$ & Hypothetical protein & XP_001912175 & Podospora anserina & $2.00 E-12$ \\
CICE8 & JK998676 & 299 & $1 / 5$ & Fasciclin domain protein & EFQ34995 & Glomerella graminicola & $5.00 E-85$ \\
CICE9 & JK998677 & 133 & $1 / 1$ & - & - & Colletotrichum lentis & - \\
CICE10 & JK998678 & 204 & $0 / 0$ & Hypothetical protein & XP_003002566 & Verticillium albo-atrum & $3.00 E-44$ \\
CICE11 & JK998679 & 129 & $0 / 2$ & Hypothetical protein & EFQ28429 & Glomerella graminicola & $6.00 E-52$ \\
CICE12 & JK998680 & 112 & $0 / 0$ & Hypothetical protein & EFQ27407 & Glomerella graminicola & $1.00 E-56$ \\
CICE14 & JK998682 & 158 & $0 / 6$ & Laccase-1 precursor & CBY01468 & Leptosphaeria maculans & $7.00 E-04$ \\
CICE15 & JK998683 & 153 & $1 / 0$ & - & - & Colletotrichum lentis & - \\
CICE18 & JK998686 & 85 & $0 / 0$ & Hypothetical protein & ELA28866 & Colletotrichum gloeosporiodes & $5.00 E-20$ \\
\hline
\end{tabular}

These effectors were identified from a directional cDNA plasmid library from leaf tissues of Lens culinaris cv. Eston infected with C. lentis isolate CT-21 undergoing the morphogenetic biotrophy-necrotrophy transition

precursor (ClCE14) with $E$ value $\leq 1 \mathrm{e}-6$. Mcl1 (Metarhizium collagen-like protein 1) acts as an antiadhesive protective coat by camouflaging antigenic structural components of the cell wall, such as $\beta$-glucans [29]. Fungal fasciclin domain-containing proteins are cell surface proteins and known to attach to the exterior leaflet of the plasma membrane by glycosylphosphatidylinositol (GPI) anchors [30]; however, ClCE8 lacks a GPI anchor addition site in its predicted peptide sequence. Laccases (EC 1.10.3.2, p-diphenol: dioxygen oxidoreductase) are copper-oxidoreductases that catalyze the biological oxidation-reduction of polyphenols with a concomitant reduction of molecular oxygen to water [31].

\section{Candidate effectors of $C$. lentis show striking similarity to those of other Colletotrichum species}

Effectors have long been regarded as a relatively species-specific repertoire of arms that dismantles resistance evolved or introgressed in host plant species [32], hence the likelihood of finding homologs in other species has been considered low. Exploiting the availability of an increasing number of fungal genomes, putative effectors were mined here at a global scale in $C$. lentis and other species with particular emphasis on the four available genomes in the genus Colletotrichum to identify and compare homologs to $C$. lentis putative effectors. With a stringent $E$ value of 1 e-50, we identified potential homologs of 10 out of 15 C. lentis candidate effectors. Top hits were found in Colletotrichum spp. and used in comparative pairwise sequence analysis. A Circos plot [33] depicting 25 ideograms representing 15 candidate effectors from $C$. lentis and 10 potential homologs from C. higginsianum, C. sublineola and C. fioriniae was generated to visualize the percentage peptide sequence similarities between candidate effectors and their homologs (Fig. 1). Covering over $80 \%$ of the effector sequences, these homologs show more than $65 \%$ peptide sequence identity, suggesting that effectors of different, but related species are not as unique as previously thought and can display significant homology across species.

Time-course expression profiling of candidate effectors Using RT-qPCR, expression of candidate effectors in planta was quantified at appressorium penetration (24 hai), during the biotrophic (44 hai) and necrotrophic phase (72 hai), as well as in mycelia and ungerminated conidia. The transcription levels of candidate effectors were normalized to the $C$. lentis house-keeping gene actin, and were expressed as relative values with 1 corresponding to expression level in mycelia.

Nine out of 14 candidate effector genes showed upregulation in their expression during in planta infection whereas the remaining five genes were repressed during plant infection (Fig. 2). The $\mathrm{C}_{\mathrm{T}}$ value for the effector gene ClCE14 was higher $\left(C_{T}>35\right)$ and therefore was not further analyzed. Three expression waves were noticeable among genes induced during infection: Appressorium penetration-specific (ClCE7, ClCE8 and ClCE11), biotrophy-specific (ClCE18) and necrotrophyspecific (ClCE1 and $C l C E 3$ through 6). Two candidate effectors (ClCE2 and ClCE9) showed conidia-specific expression. 


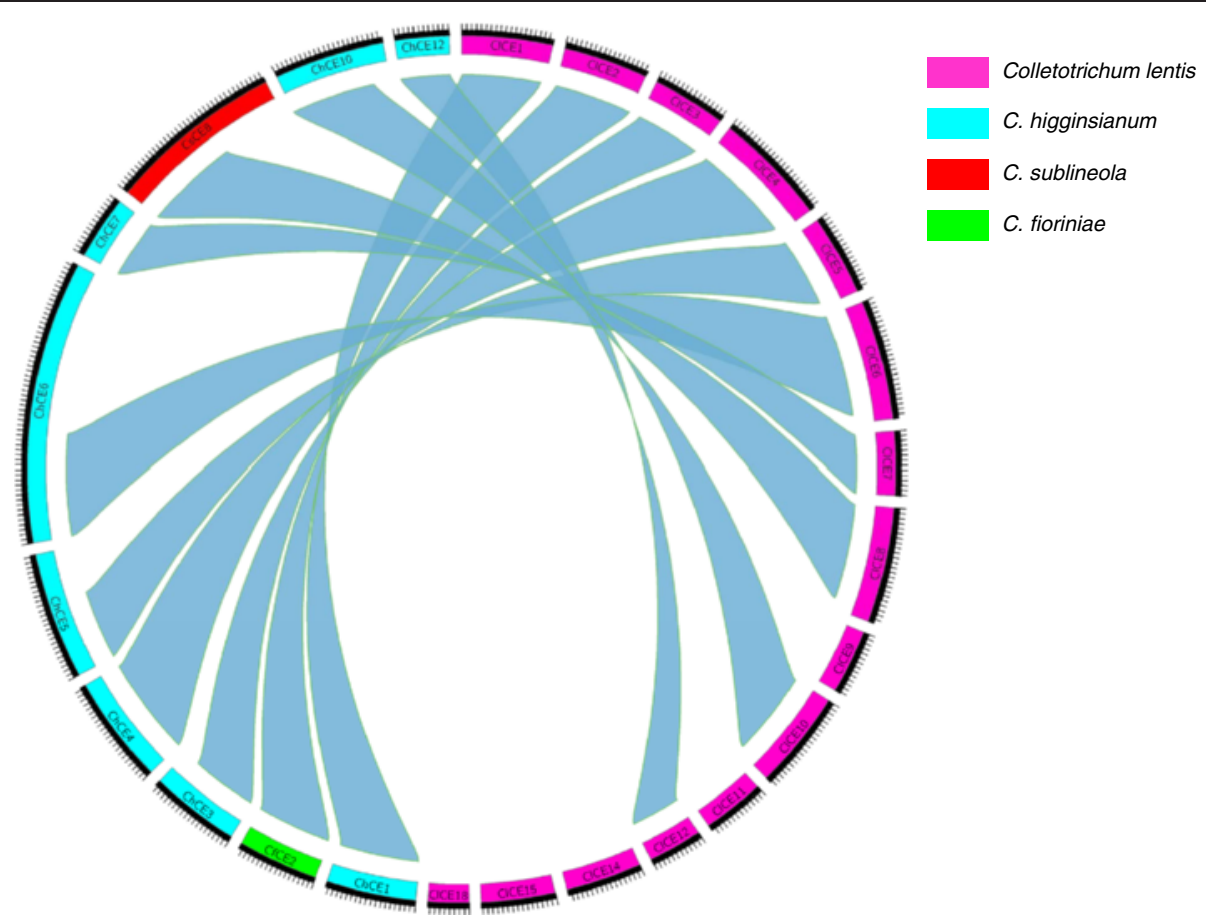

Fig. 1 Comparative analysis of Colletotrichum lentis candidate effectors with other Colletotrichum spp. The Circos plot shows candidate effectors as ideograms. Stringent $E$ value 1 e-50 was used to identify potential homologs and only top BLAST hit was included in the Circos plot. Ribbon links convey the linked region between two ideograms with over $65 \%$ amino acid residue identities

\section{Effector polymorphism in C. lentis isolates collected from western Canada}

Considering the hyper-variable nature of effectors, we scanned all 15 candidate effectors against the draft genome of C. lentis isolate CT-30 (race 0) and identified two silent SNPs (Race 1/Race 0, T/C) in the two candidate effectors ClCE6 and ClCE8. ClCE6 is a hypothetical protein whereas ClCE8 contains a fasciclin domain (pfam02469). With a stringent $E$ value of 1e-50, six homologs of ClCE8 belonging to C. sublineola, C. fioriniae, C. graminicola, $C$. gloeosporioides, C. higginsianum and C. orbiculare were identified in the NCBInr protein database. All six homologs contain a putative 17-aa SP for potential secretion in host plants and a fasciclin domain (variable in length) for function. ClCE8 homologs are relatively large proteins (>300 aa) except for the C. higginsianum fasciclin protein (Chfas [CCF47579], 148-aa), suggesting ClCE8 and Chfas are likely splicing variants. ClCE8 homologs contain conserved amino acid residue blocks (in red in Fig. 3a) in the fasciclin domain, indicating functional homology among homologs, and most variability is seen at the C-terminus. Fungal fasciclin proteins are known to function as virulence factors [34].

ClCE8 homologs were retrieved from the NCBInr protein database using a stringent $E$ value of 1e-50. A Neighbor-Joining phylogram of ClCE8 homologs was generated using a Poisson-correction model $(K=-\ln (1-D))$. ClCE8 and Chfas from C. higginsianum formed a cluster separate from the remaining six fasciclin proteins with lowest mean number of amino acid substitution(s) per site $(\mathrm{K}=0.02)$ between them, suggesting that both putative effectors are likely functional homologs (Fig. 3b). Considering the small size $(<300$ aa), both ClCE8 and Chfas are likely delivered in host plants by their respective pathogens to facilitate fungal colonization.

To test whether SNPs in ClCE6 and ClCE8 can differentiate virulent race 0 from less virulent race 1 isolates, 52 C. lentis isolates collected from western Canada were randomly selected and phenotyped using the lentil differential cultivar CDC Robin with partial resistance to race 1 , but not to race 0 isolates. Thirty nine out of 52 isolates were fully pathogenic on CDC Robin and therefore classified as race 0 isolates. The remaining 13 isolates caused significantly fewer lesions on the cultivar, hence were classified as race 1 isolates. KASPar assays revealed that the ClCE6 KASPar marker could differentiate race 0 from race 1 isolates based on a correlation of $100 \%$ between genotypic and phenotypic assay results. In contrast, the ClCE 8 KASPar marker identified six race 1 isolates as race 0 . Results suggest that ClCE6 is likely co-segregated with loci governing the virulence of $C$. lentis (Table 2, Figs. 4 and 5). 


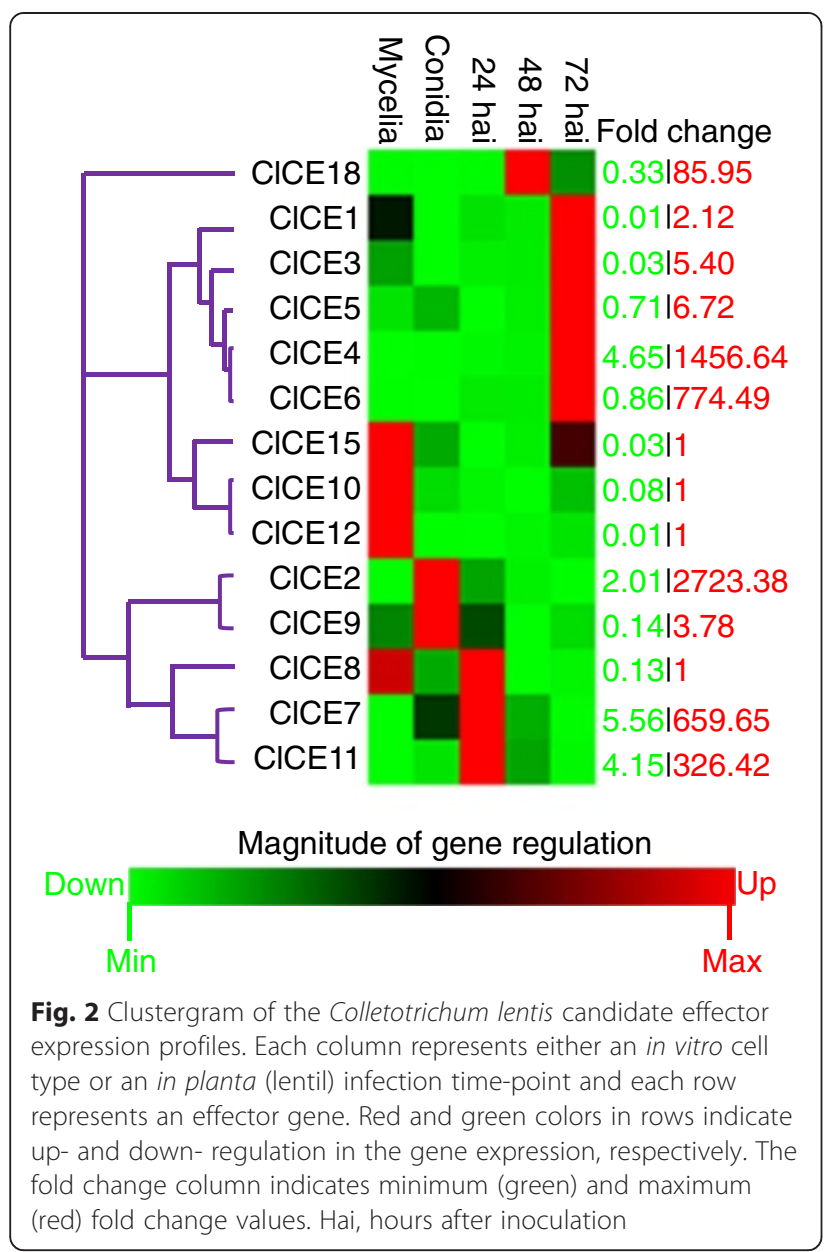

\section{CIToxB is likely a host-specific toxin and implicated in virulence differentiation of $C$. lentis races}

In a previous study on EST mining of $C$. lentis infected lentil tissues, ClToxB (GenBank accession JZ350031) was identified [5]. Unlike PtrToxB of P. tritici-repentis, only one copy of $C l T o x B$ was detected in the $C$. lentis genome (unpublished data). The full length cDNA of ClToxB was sequenced here revealing an open reading frame of 282-bp with a 27-bp 5-prime untranslated region (UTR) and a 218-bp 3-prime UTR that encodes a pre-protein of 94-aa. An SP of 19-aa with a cleavage site in-between alanine-19 and glutamate-20 was predicted in the preprotein, and four cysteine residues were found in the mature protein (75-aa) (Fig. 6). ClToxB is a small (94 aa), stable (instability index 20.99), soluble (lacks transmembrane helix, N/O-glycosylation and GPI addition sites) and acidic protein ( $\mathrm{p} I 5.52)$ protein with $7.85 \mathrm{KDa}$ molecular weight (Table 3 ). Using the non-redundant protein database available at the NCBI with an $E$ value cut-off of 1e-6, homologs of ClToxB were identified in C. higginsianum, C. orbiculare, C. gloeosporiodes, and Magnaporthe oryzae. Multiple sequence alignment with ClustalW revealed extensive similarity with PtrToxB including that of the most virulent isolate alg3-24 (Fig. 7a, Table 4). ClToxB is a conserved protein with 4 characteristic cysteine residues. Using the DiANNA web server, two disulfide bonds were predicted in the mature protein, which may provide stability to ClToxB in the host apoplast (Fig. 7b), and RTqPCR analysis showed that the expression of ClToxB peaked during the biotrophy-necrotrophy switch (48 hpi), suggesting a potential role in anthracnose development. Expression polymorphism was detected at $48 \mathrm{hpi}$ as the $C l T o x B$ transcript level was higher in lentil tissues infected with the more virulent isolate CT-30 (race 0) than that inoculated with CT-21 (race 1) (Fig. 8). This differential transcript level may be associated with virulence patterns of $C$. lentis races. To investigate whether $C l T o x B$ also shows sequence polymorphism, the full length cDNA from isolate CT-21 (race 1) was mapped onto the CT30 (race 0) draft genome. No DNA polymorphism was detected between the two isolates representing races 1 and 0 .

To confirm whether ClToxB is a host-specific toxin, ClToxB with and without SP were expressed in tobacco via agroinfiltration. Phytophthora infestans infestin 1 [35] used as a positive control in the transient expression assay caused confluent cell death at 3 days postinfiltration (dpi) whereas that was not the case for ClToxB though scattered cell death flecks were found in the vicinity of the agroinfiltration site. No chlorosis was observed in the zones infiltrated with $\mathrm{ClToxB}$ and ClToxB $\triangle \mathrm{SP}$, suggesting that ClToxB is likely a hostspecific toxin (Fig. 9).

Taken together, data indicate that unlike in the case of PtrToxB in P. tritici-repentis, presence or absence of $C l T o x B$ or sequence polymorphisms do not determine the races that have been described in $C$. lentis. However, $C l T o x B$ may contribute to the virulence profile of races on lentil through differences in the level of expression, thereby amplifying cell death signals at the biotrophynecrotrophy switch.

\section{CIToxB is unlikely a foreign gene in the C. lentis genome}

Comparison of molecular tree topologies generated based on multiple loci (species tree) (Fig. 10a) and based on the sequences of ToxB homologs (Fig. 10b) revealed incongruency between the trees, which would be expected in case of HGT. No patchy phyletic distribution of ToxB was found in the gene tree, and ToxB genes from closely related species like Colletotrichum spp. were grouped together as a clade. A 14100-bp fragment containing the ClToxB locus (Scaffold_10) retrieved from our newly assembled $C$. lentis draft genome revealed an average of 51.08 and $56.84 \mathrm{GC} \%$ for the scaffold and the ClToxB gene, respectively, which is not atypical as would be expected in cases of HGTs (Fig. 11a). Genes involved in HGTs are unlikely to be present in closely related species, 


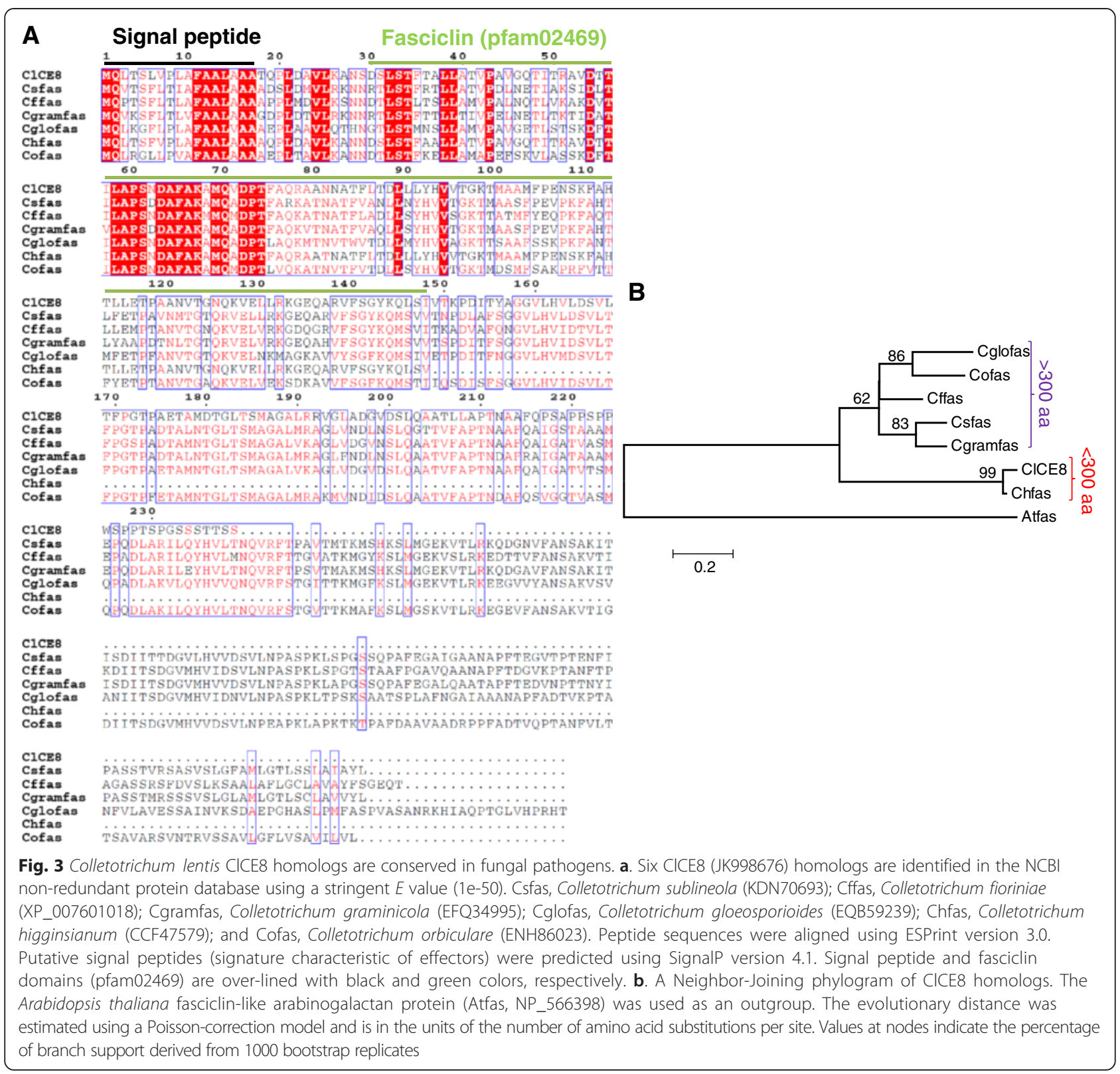

and if present, do not show syntenic relationship as events of horizontally transferred genes are random rather than targeted. To confirm this hypothesis, a MUMmerplot was generated using NUCmer alignment of the ToxB loci contained in Scaffold_10 (5500-8000 bp) from C. lentis and Supercontig_1.8152 (1-2079 bp) from C. higginsianum. $C l T o x B$ and $C h T o x B$ were located in the conserved syntenic block (Fig. 11b), indicating that $C l T o x B$ was not acquired by HGT. Recently transferred foreign genes, especially those from prokaryotes, show different preferential codon usage pattern compared to the native genes as they have yet to adapt to their new recipient host genomes. Comparison of the codon usage profile of ClToxB with the native house-keeping gene actin $(C l A C T)$ and a previously characterized biotrophy-necrotrophy switch regulator CINUDIX [27] showed no atypical variation in profiles (Fig. 11c). Taken together, our data confirm that $C l T o x B$ is a native rather than foreign gene in the C. lentis genome.

\section{CIToxB is a virulence factor}

An RNA-silencing approach was used to determine the functional role of ToxB in $C$. lentis. The $C l T o x B$ was cloned into a dual promoter silencing vector pSilentDual 1 to generate sense and antisense ClToxB RNA pools. The vector pSilent-Dual 1-ClToxB was then used to transform $C$. lentis spheroplasts (Fig. 12a). Fifteen transformants were retrieved from this transformation 
Table 2 Race indexing of Colletotrichum lentis isolates based on phenotyping and genotyping

\begin{tabular}{|c|c|c|c|}
\hline \multirow[t]{2}{*}{ Isolates } & \multicolumn{3}{|c|}{ Race indexing } \\
\hline & Phenotype & ClCE6 & CICE8 \\
\hline $\mathrm{Cl}-11$ & 0 & 0 & 0 \\
\hline $\mathrm{Cl}-15$ & 1 & 1 & 1 \\
\hline $\mathrm{Cl}-16$ & 0 & 0 & 0 \\
\hline $\mathrm{Cl}-17$ & 1 & 1 & 1 \\
\hline $\mathrm{Cl}-20$ & 0 & 0 & 0 \\
\hline $\mathrm{Cl}-21$ & 1 & 1 & 1 \\
\hline $\mathrm{Cl}-23$ & 0 & 0 & 0 \\
\hline $\mathrm{Cl}-26$ & 0 & 0 & 0 \\
\hline $\mathrm{Cl}-28$ & 1 & 1 & 1 \\
\hline $\mathrm{Cl}-29$ & 1 & 1 & 1 \\
\hline $\mathrm{Cl}-30$ & 0 & 0 & 0 \\
\hline $\mathrm{Cl}-31$ & 0 & 0 & 0 \\
\hline $\mathrm{Cl}-32$ & 0 & 0 & 0 \\
\hline $\mathrm{Cl}-33$ & 1 & 1 & 0 \\
\hline $\mathrm{Cl}-34$ & 0 & 0 & 0 \\
\hline $\mathrm{Cl}-35$ & 1 & 1 & 0 \\
\hline $\mathrm{Cl}-37$ & 0 & 0 & 0 \\
\hline $\mathrm{Cl}-38$ & 0 & 0 & 0 \\
\hline $\mathrm{Cl}-39$ & 1 & 1 & 0 \\
\hline $\mathrm{Cl}-43$ & 1 & 1 & 1 \\
\hline $\mathrm{Cl}-44$ & 0 & 0 & 0 \\
\hline $\mathrm{Cl}-45$ & 0 & 0 & 0 \\
\hline $\mathrm{Cl}-46$ & 0 & 0 & 0 \\
\hline $\mathrm{Cl}-47$ & 0 & 0 & 0 \\
\hline $\mathrm{Cl}-58$ & 1 & 1 & 1 \\
\hline $\mathrm{Cl}-59$ & 0 & 0 & 0 \\
\hline $\mathrm{Cl}-60$ & 0 & 0 & 0 \\
\hline $\mathrm{Cl}-181$ & 0 & 0 & 0 \\
\hline $\mathrm{Cl}-185$ & 0 & 0 & 0 \\
\hline $\mathrm{Cl}-187$ & 0 & 0 & 0 \\
\hline $\mathrm{Cl}-188$ & 0 & 0 & 0 \\
\hline $\mathrm{Cl}-189$ & 0 & 0 & 0 \\
\hline $\mathrm{Cl}-190$ & 0 & 0 & 0 \\
\hline Cl-191 & 0 & 0 & 0 \\
\hline Cl-192 & 0 & 0 & 0 \\
\hline $\mathrm{Cl}-209$ & 0 & 0 & 0 \\
\hline $\mathrm{Cl}-231$ & 0 & 0 & 0 \\
\hline $\mathrm{Cl}-233$ & 0 & 0 & 0 \\
\hline $\mathrm{Cl}-255$ & 0 & 0 & 0 \\
\hline $\mathrm{Cl}-364$ & 0 & 0 & 0 \\
\hline $\mathrm{Cl}-366$ & 0 & 0 & 0 \\
\hline $\mathrm{Cl}-368$ & 0 & 0 & 0 \\
\hline
\end{tabular}

Table 2 Race indexing of Colletotrichum lentis isolates based on phenotyping and genotyping (Continued)

\begin{tabular}{llll}
\hline $\mathrm{Cl}-379$ & 0 & 0 & 0 \\
$\mathrm{Cl}-380$ & 0 & 0 & 0 \\
$\mathrm{Cl}-383$ & 0 & 0 & 0 \\
$\mathrm{Cl}-384$ & 1 & 1 & 0 \\
$\mathrm{Cl}-397$ & 0 & 0 & 0 \\
$\mathrm{Cl}-400$ & 1 & 1 & 0 \\
$\mathrm{Cl}-402$ & 1 & 1 & 0 \\
$\mathrm{Cl}-417$ & 0 & 0 & 0 \\
$\mathrm{Cl}-418$ & 0 & 0 & 0 \\
$\mathrm{Cl}-422$ & 0 & 0 & 0 \\
\hline
\end{tabular}

Differential Lens culinaris Cv. CDC Robin, and CICE6 and CICE8 KASPar markers were used to phenotype and genotype $C$. lentis isolates, respectively

and subjected to RT-qPCR. No knock down-penalty was observed among silenced strains as they grew normally and conidiogenesis was similar to the wild-type isolate CT-21. Three silenced strains were selected for virulence/pathogenicity testing. Among them was the strain SToxB-8, which displayed only $5 \%$ of the wild-type ClToxB expression (Fig. 12b). Susceptible lentil cultivar Eston was used to evaluate the virulence of silenced strains. Lentil plants infected with the silenced strain SToxB-8 showed significantly reduced anthracnose symptoms (disease severity $\pm \mathrm{SE}$ of $13.08 \pm 1.65$ ) compared to the wild-type strain CT-21 (disease severity \pm SE of $87.5 \pm 1.58$ ) (Fig. 12c). To investigate which stage of fungal development was impaired by the silencing of $C l T o x B$, infected leaf tissues were collected at 3 days after inoculation (dai) and visualized under a light microscope. Quantitative variation in virulence was observed during in planta fungal proliferation. The silenced strain SToxB with the lowest level of $C l T o x B$ expression caused fewer anthracnose lesions at 6 dai (Fig. 12c), which was likely associated with the delayed biotrophy-necrotrophy switch as can be seen in case of the SToxB-8 at 3 dai (Fig. 12d).

\section{CIArg is a foreign gene in the genus Colletotrichum}

ESTs were further scanned for potential (inter kingdom) HGT events using the BLASTX algorithm on Linux standalone BLAST and NCBInr protein database. We hypothesized that the top hits of a HGT candidate with an $E$ value 1e-50 should match to distantly related fungal species (in case of intra-kingdom HGT) or bacterial species (in case of inter-kingdom HGT). Only one EST met this criterion and the full length coding sequence (CDS) was retrieved by mapping the EST onto the $C$. lentis draft genome. When the CDS was queried against NCBInr protein database with an $E$ value 1e-50 and maximum target sequences 100, the top seven hits were matched to six Colletotrichum spp. (with an $E$ value 0 and $95-99 \%$ query 


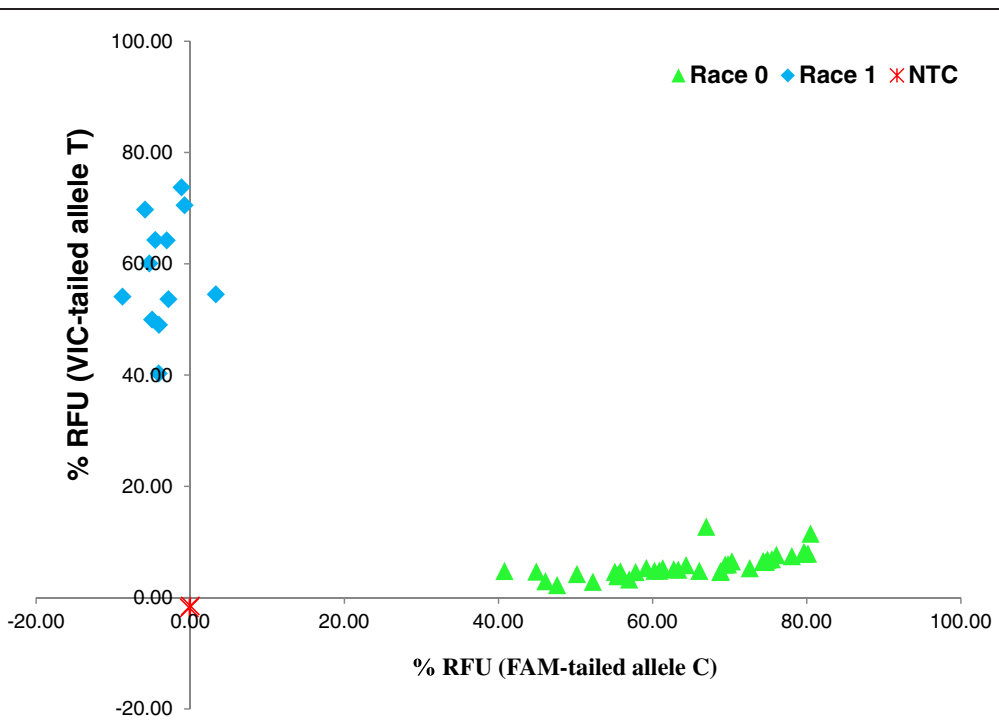

Fig. 4 Allele discrimination plot based on percentage endpoint fluorescence (RFU) in KASPar assays. Fifty two Colletotrichum lentis isolates collected from western Canada were genotyped using the CICE6 KASPar marker. NTC: No Template Control

coverage) and the remaining 93 hits were matched to the kingdom Prokaryota/Bacteria (68 hits with an $E$ value 0 and $89-97 \%$ query coverage). The top ten hits are listed in the Table 5. All hits were identified as argininosuccinate lyase (Arg; EC 4.3.2.1) that is implicated in the urea cycle. Arg catalyzes a reaction that forms fumarate and arginine from $\mathrm{L}$-argininosuccinate. ClArg is located in a block on scaffold_5 of the C. lentis draft genome that is syntenic with a block on supercontig_1.321 of the closely related species $C$. higginsianum (Fig. 13a), suggesting that $\mathrm{Arg}$ was horizontally transferred into a common ancestor of the genus Colletotrichum. ClArg contains no intron, and has a relatively higher GC content $(63.25 \%$, Fig. 13b) compared to the rest of the genome, and atypical codon usage compared to eukaryotic genes ClACT and ClNUDIX (Fig. 13c), further confirming that ClArg is not a native gene in the C. lenis genome.

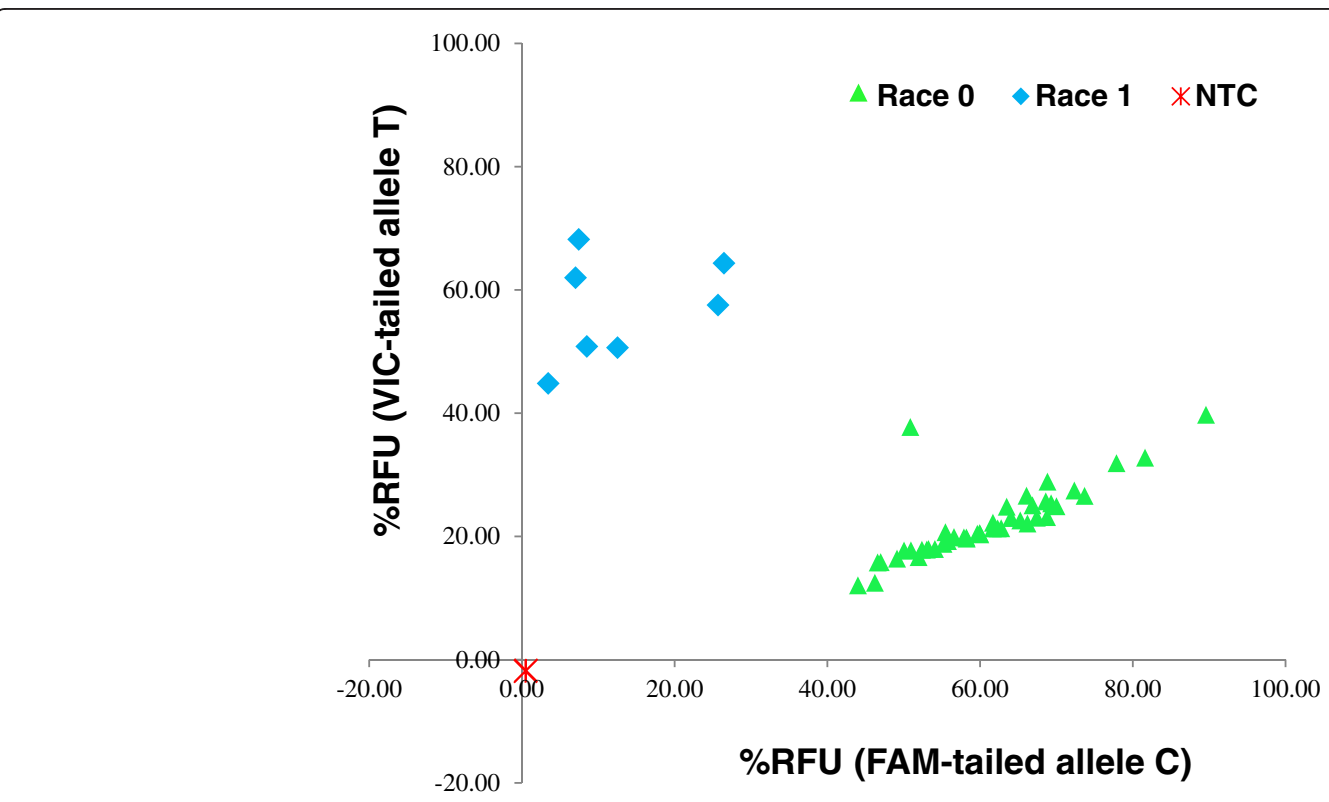

Fig. 5 Allele discrimination plot based on percentage endpoint fluorescence (RFU) in KASPar assays. Fifty two Colletotrichum lentis isolates collected from western Canada were genotyped using CICE8 KASPar marker. NTC: No Template Control 


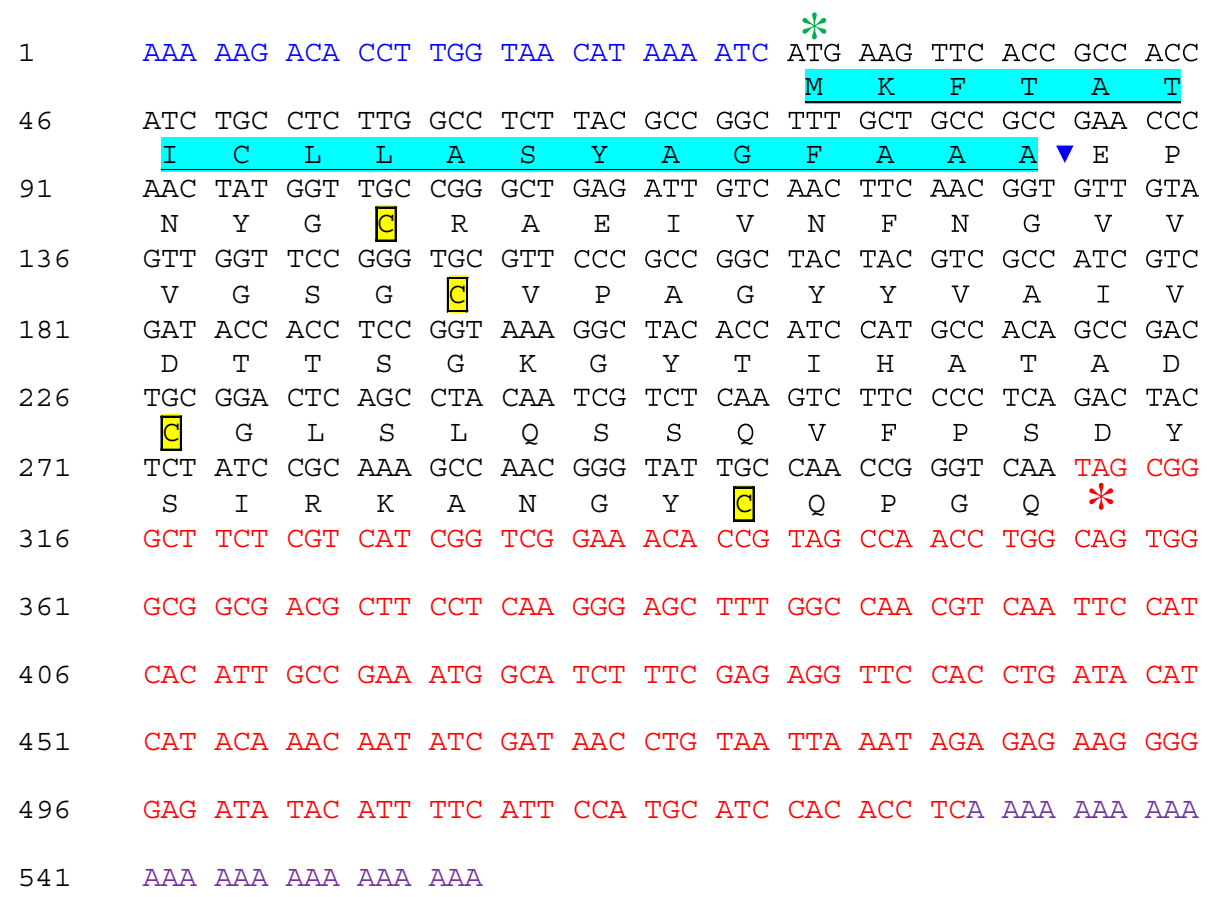

Fig. 6 Colletotrichum lentis CIToxB cDNA sequence. Green and red asterisks indicate start and stop codons. Signal peptide for potential secretion is highlighted in turquoise color. Cysteine residues are highlighted and boxed

\section{Discussion}

Expression of pathogen effectors in plants is a perfect example of the "extended phenotype", a concept put forward by Richard Dawkins [36] in his classic book "Extended phenotype: The long reach of genes". Effector biology of filamentous fungi pathogenic to economically important crops is an established field in plant pathology. Since the introduction of next-generation sequencing technologies, the number of sequenced fungal genomes and transcriptomes has been increasing exponentially, and as a result, the discovery of effector genes is accelerating.

Table 3 CIToxB biochemical properties predicted through in silico analyses

\begin{tabular}{lll}
\hline Characteristics & Value & Softwares \\
\hline Signal peptide & 1 & SignalP v4.1 \& iPSORT \\
Cysteine residues & 4 & Mannual counting \\
Cystine bridges & 2 & DiANNA v1.1 \\
Isoelectric point (pl) & 5.52 & ExPASy Compute PI/Mw \\
Molecular weight & 7.85 KDa & ExPASy Compute PI/Mw \\
Transmembrane helix & 0 & TMHMM v2.0 \\
GPI addition site & 0 & Big-PI Fungal Predictor v3.0 \\
N-glycosylation site(s) & 0 & NetNGlyc 1.0 \\
O-glycosylation site(s) & 0 & NetOGlyc v3.1 \\
Grand average of hydropathicity & -0.087 & ProtParam \\
Instability index & 20.99 & ProtParam \\
\hline
\end{tabular}

Effector biology has practical implications for resistance breeding via effector-assisted selection of plants in populations segregating for disease resistance [37-39]. The role of effectors in fungal pathogenicity and virulence is well established, and thus monitoring allelic diversity of effectors in evolving pathogen population and among races can assist in $R$ gene deployment in cultivated varieties $[38,39]$. Therefore, generating an inventory of candidate effector genes is the first step towards their potential usage in disease resistance breeding in economically important food crops, such as cereals, oilseeds and legumes. Comparison of effector gene structure and function among closely and more distantly related species, now increasingly more feasible as the number of sequenced genomes is increasing rapidly, will also shed new light on the effects of selection on population divergence and speciation, as well as the frequency of HGT events.

We identified 15 candidate effectors of $C$. lentis mined from the biotrophy-necrotrophy switch-specific cDNA library developed previously with the objective to identify effectors that may be involved in the virulence of $C$. lentis on its host lentil, and may differentiate between the two pathogenic races described in the western Canadian population of this pathogen. Among them were two C. lentis - specific effectors (ClCE9 and ClCE15). For the majority of candidate effectors (8), the top BLAST hit ( $E$ value $\leq 1 \mathrm{e}-6)$ was matched to fungal proteins within the genus Colletotrichum predicted from automated 


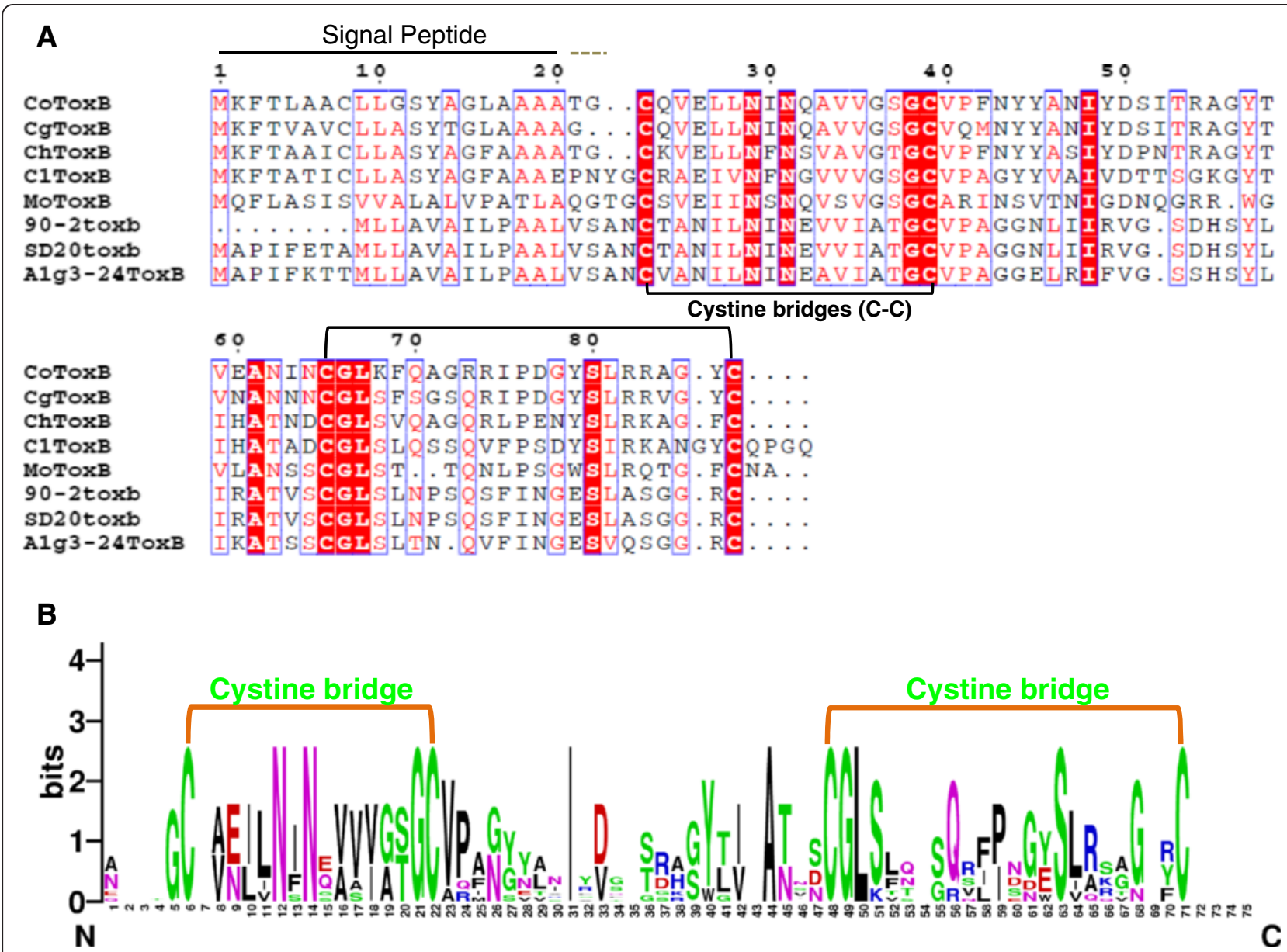

Fig. 7 Structural similarity of Colletotrichum lentis CIToxB with ToxB of other species. a. CIToxB shows structural similarity to ToxB proteins from closely related species and 3 ToxB variants from Pyrenophora tritici-repentis. Four conserved cysteine residues were identified in the mature protein, and these amino acid residues are likely to form two cystine bridges, which may provide stability to ToxB proteins in the host cell apoplast. Peptide sequences were aligned using ESPrint version 3.0. Putative signal peptides (black line) were predicted using SignalP version 4.1. CIToxB, Colletotrichum lentis (Race 1, CT-21 [less virulent isolate] and Race 0, CT-30 [virulent isolate]); CoToxB, C. orbiculare 104-T (virulent isolate); CgToxB, C. gloeosporiodes Nara gc5 (virulent isolate); ChToxB, Colletotrichum higginsianum IMI349063 (virulent isolate); MoToxB, Magnaporthe oryzae Y34 (virulent isolate); 90-2toxb, Pyrenophora tritici-repentis 90-2 (Race 4, 90-2 [avirulent isolate]); SD20toxb, Pyrenophora tritici-repentis (Race 4, SD20 [avirulent isolate]); and Alg3-24ToxB, Pyrenophora tritici-repentis (Race 5, Alg3-24 [most virulent isolate]). b. CIToxB homologs contain a conserved cysteine residue pattern. Sequence logo was generated from the Clustal W-aligned ToxB mature proteins using Seg2Logo (Thomsen and Nielsen [50]). Big and small amino acid residue stacks indicate conserved and variable sites, respectively. Conserved cysteine residues are likely to form two cystine bridges (disulfide bonds)

Table 4 CIToxB homologs

\begin{tabular}{|c|c|c|c|c|c|c|c|}
\hline Toxin B homologs & Pathogen & GenBank & Isolate/Strain & Peptide (aa) & $\mathrm{SP}^{\mathrm{a}}$ & $M P^{b}$ & Identity |Similarity ${ }^{c}$ \\
\hline CIToxB & Colletotrichum lentis & $J Z 350031$ & CT-21 (Race 1) \& CT-30 (Race 0) & 94 & 19 & 75 & $100 \mid 100$ \\
\hline Ptrtoxb & Pyrenophora tritici-repentis & AAM00019 & SD20 (Race 4) & 88 & 23 & 65 & $40 \mid 50$ \\
\hline PtrToxB & Pyrenophora tritici-repentis & AF483831 & Alg3-24 (Race 5) & 87 & 23 & 64 & $39 \mid 53$ \\
\hline CgToxB & Colletotrichum gloeosporiodes & ELA28482 & Nara gc5 & 86 & 19 & 67 & 44 | 57 \\
\hline ChToxB & Colletotrichm higginsianum & CCF45936 & IMI349063 & 87 & 19 & 68 & $52 \mid 66$ \\
\hline CoToxB & Colletotrichum orbiculare & ENH84621 & 104- $\mathrm{T}$ & 87 & 19 & 68 & $44 \mid 58$ \\
\hline MoToxB & Magnaporthe oryzae & ELQ42910 & Y34 & 88 & 20 & 68 & $36 \mid 52$ \\
\hline Ptrtoxb & Pyrenophora tritici-repentis & AF483832 & 90-2 (Race 4) & 80 & 15 & 65 & $40 \mid 50$ \\
\hline
\end{tabular}

An $E$ value cut-off of 1e-6 was used to extract homologs of CIToxB from NCBInr protein database. All homologs contain four characteristic cystine residues, which are likely to form 2 disulfide bonds. ${ }^{a}$ SP, Signal Peptide (predicted using SignalP server version 4.1) ${ }^{\mathrm{b}} \mathrm{MP}$, Mature Protein ${ }^{\mathrm{c}}$ Identity/Similarity (in per cent) as calculated by Needleman-Wunsch pairwise alignment of CIToxB (MP) with its homologs. 


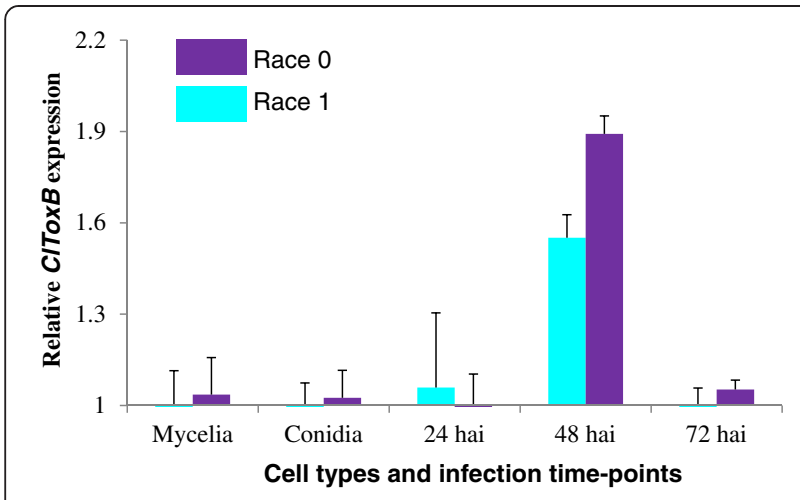

Fig. 8 Comparative expression profiling of Colletotrichum lentis CITOXB. X-axis shows two fungal cell types (mycelia and conidia) and three in planta infection time-points (24 hai, 48 hai and 72 hai) whereas $Y$-axis shows relative gene expression on a $\log 2$ scale. Hai: Hours after inoculation

whole-genome sequencing and annotation projects (Fig. 1). Only three candidate effectors had detectable homologs with putative functions. The majority of identified candidate effectors (8) are in planta-induced, but during different stages of lentil infection, namely appressorium penetration, biotrophic and necrotrophic phases. The expression of the remaining candidate effectors displayed no significant alternation between in vitro vegetative growth and in planta colonization (Fig. 2). This suggests that $C$. lentis deploys different sets of effector proteins to condition and promote virulence.

Putative functions were assigned to 3 candidate effectors based on orthology to proteins of known functions (Table 1). Effector ClCE5, a Mcl1 homolog showed a 6.7fold induction during the necrotrophic phase (Fig. 2). Mcl1 was originally identified in the entomopathogenic fungus Metarhizium anisopliae and was shown to be expressed within 20 min of contact between the pathogen and the hemolymph of the lepidopteran model insect Manduca sexta. Mcl1 envelopes $M$. anisopliae hyphae, and in doing so, camouflages antigenic structures, such as $\beta$-glucan to avoid recognition by the immune system of the insect. Targeted disruption of Mcl1 resulted in reduced virulence to $M$. sexta [29]. Since the Mcl1 homolog ClCE5 showed a necrotrophy-specific transcriptional activation, it may promote in planta fungal proliferation by inducing cell death, thereby facilitating fast growth of secondary hyphae that further kill and destroy plant tissues during the necrotrophic phase. Fasciclin proteins are GPI-linked cell surface proteins that mediate cell adhesion [40]. The biological function of fasciclin (MoFLP1) was demonstrated in $M$. oryzae wherein it is involved in conidiation, adhesion of fungal structures (conidia and appressoria) on the hydrophobic surface and virulence on rice [34]. It is likely
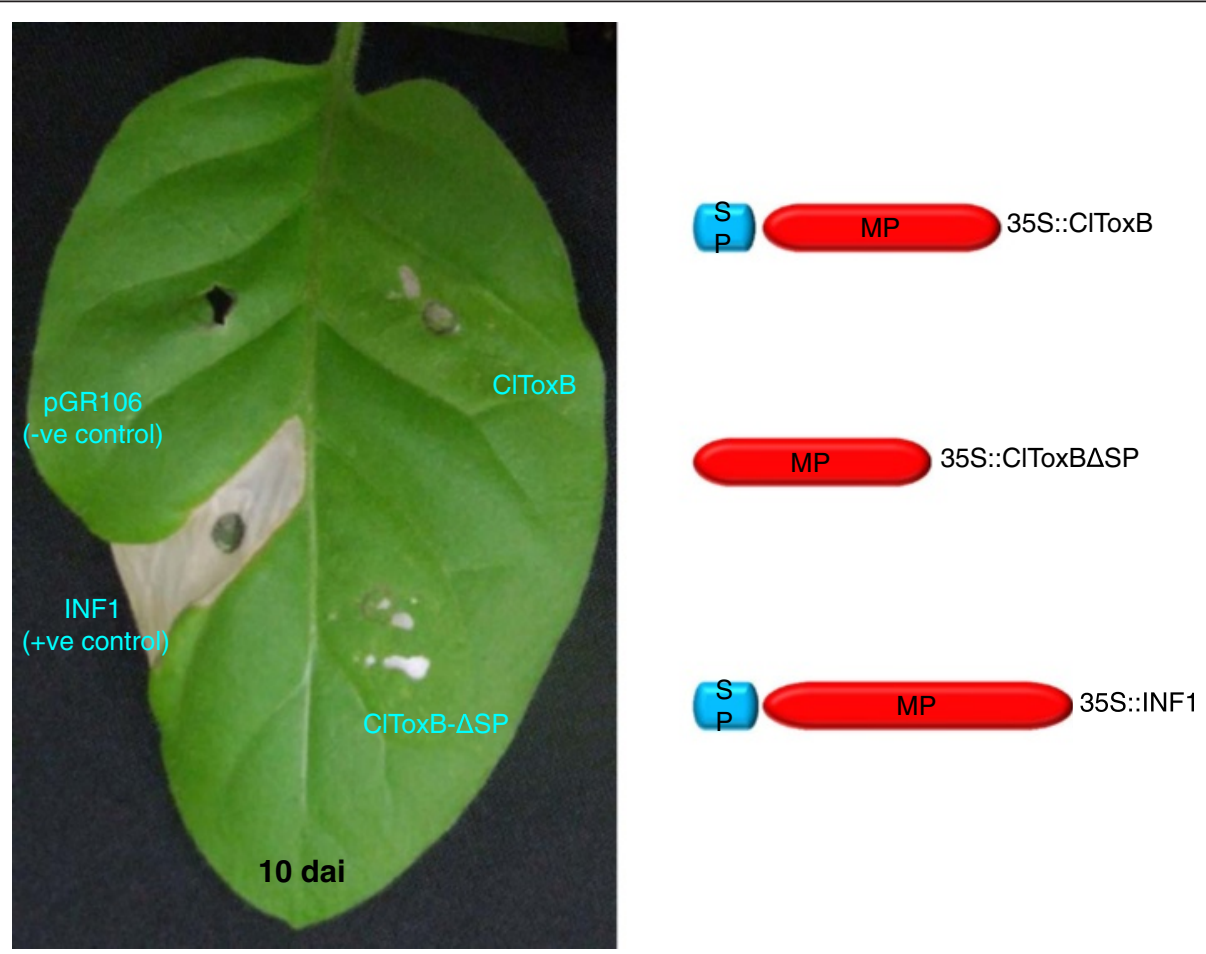

Fig. 9 CIToxB is likely a host-specific toxin. CIToxB with and without signal peptide were cloned in the PVX-based binary vector pGR106. Agrobacterium tumefaciens strains carrying pGR106-CIToxB and pGR106-CIToxB $\triangle$ SP were expressed in the model plant tobacco (Nicotiana tobacum), and macroscopic cell death/necrosis was monitored 3 through 10 days post-infiltration (dpi). Phytophthora infestans infestin 1 (INF1) elicitin and empty vector were used as positive and negative controls, respectively. SP, signal peptide and MP, mature protein 


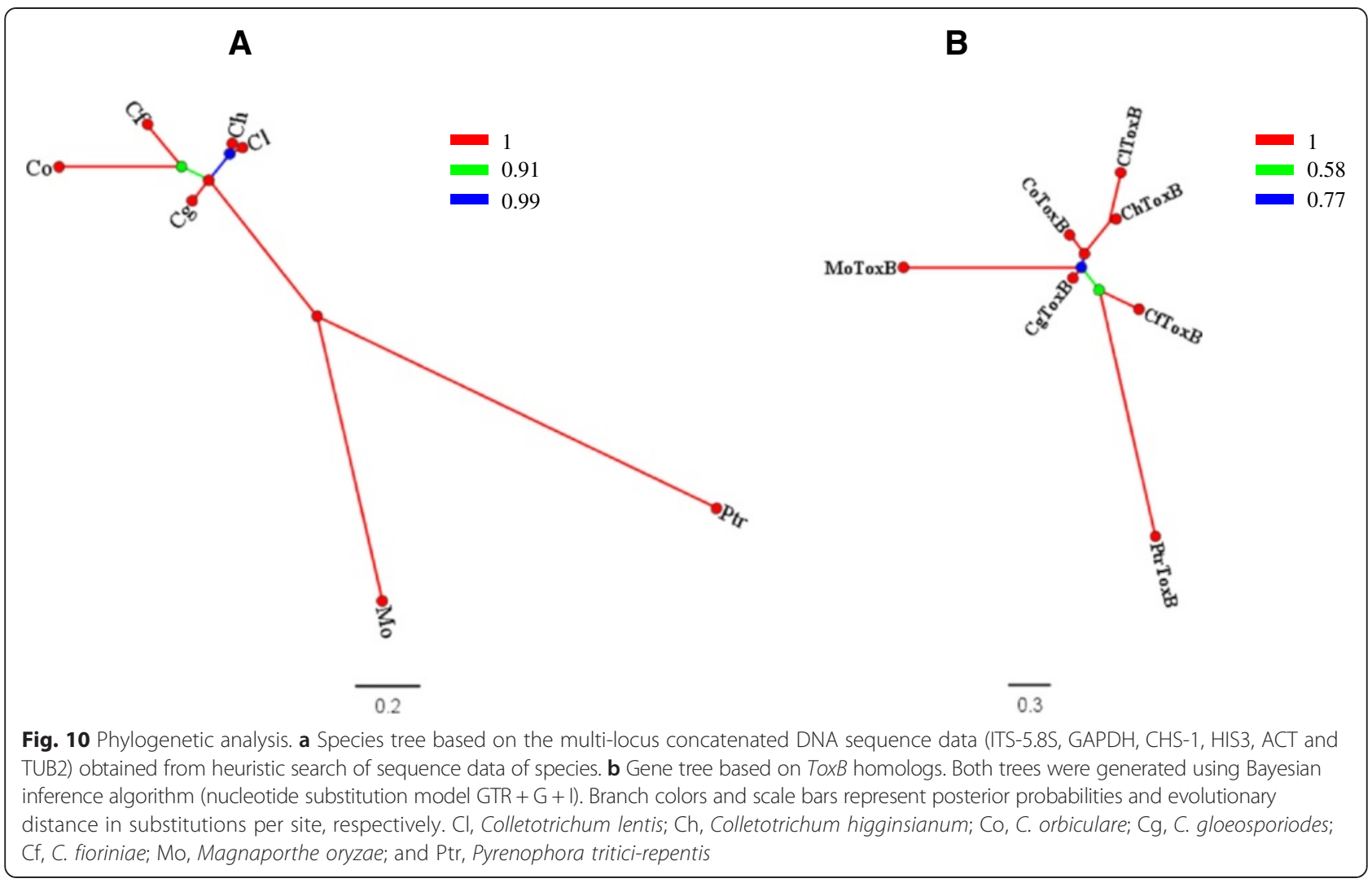

that $C$. lentis secretes the fasciclin homolog ClCE8 during conidium and appressorium adhesion on the plant surface, though it might also mediate down-stream infection related to fungal development. Recently, a secreted laccase (ClCE14 ortholog) was found to be up-regulated in appressoria formed in planta and during the biotrophic phase [3] supporting the role of fungal laccases during early fungal-plant interactions, and fungal virulence, as proposed for the chestnut blight fungus Cryphonectria parasitica [41]. However, the expression of ClCE14 was found to be quite low throughout the $C$. lentis infection process.

A Toxin B homolog ClToxB was also identified in the cDNA library [5]. Toxin $B$ is well characterized in necrotrophic pathosystems, such as $P$. tritici-repentis - wheat; however, its role in hemibiotrophic pathosystems, such

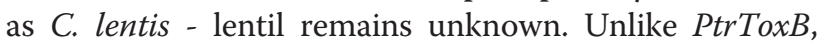
$C l T o x B$ is a single copy gene and no DNA polymorphism was found in $C l T o x B$ of the two isolates representing the two $C$. lentis races 0 and 1 . However, expression levels varied with a higher transcript level observed for the virulent race 0 isolate compared to the race 1 isolate during the switch to necrotrophy (Fig. 8). Like PtrToxB (6.6 $\mathrm{KDa}), \mathrm{ClToxB}(7.8 \mathrm{KDa})$ is likely a host (lentil)-specific toxin (Fig. 9). However, infiltration of the purified ClToxB in lentil tissues is required to confirm its role as a hostspecific toxin. Colletotrichum lentis delivers the effector protein CtNUDIX into lentil cells specifically at the biotrophy-necrotrophy switch signaling a transition in the pathogen to the anthracnose causing necrotrophic phase by causing cell death [27]. ClToxB might be involved in amplifying cell death signals during the biotrophy-necrotrophy switch and thus may contribute to quantitative differences in virulence between the $C$. lentis races 0 and 1 . To confirm the role of ToxB in C. lentis as a virulence factor, an RNAi approach was used to knock-down ClToxB mRNA levels. No growth or conidiogenesis penalty was observed among the silenced strains, which is consistent with observations in P. tritici-repentis that toxins have an exclusive role in pathogenesis. Three silenced strains displaying varying mRNA levels (5 to $80 \%$ of the wild-type strain) were used to infect Eston plants. Severity of anthracnose disease was correlated with the $C l T o x B$ transcript level as SToxB- 8 and SToxB-1 strains expressing ToxB at a rate of 5 and $80 \%$ of the wild-type, respectively, caused significantly reduced anthracnose severity on leaves and stems (13.08 and $72.24 \%$, respectively compared to $87.5 \%$ in plants infected with the wild-type isolate CT-21 at 6 dai) (Fig. 12b and c). Dot-plot analysis revealed a conserved syntenic block in the related species $C$. higginisianum and together with comparative phylogeny (species tree versus ToxB tree), GC pattern along the $\operatorname{Tox} B$ containing pseudomolecule, and codon usage profile $\operatorname{ToxB}$, was confirmed not to be a foreign gene in $C$. 


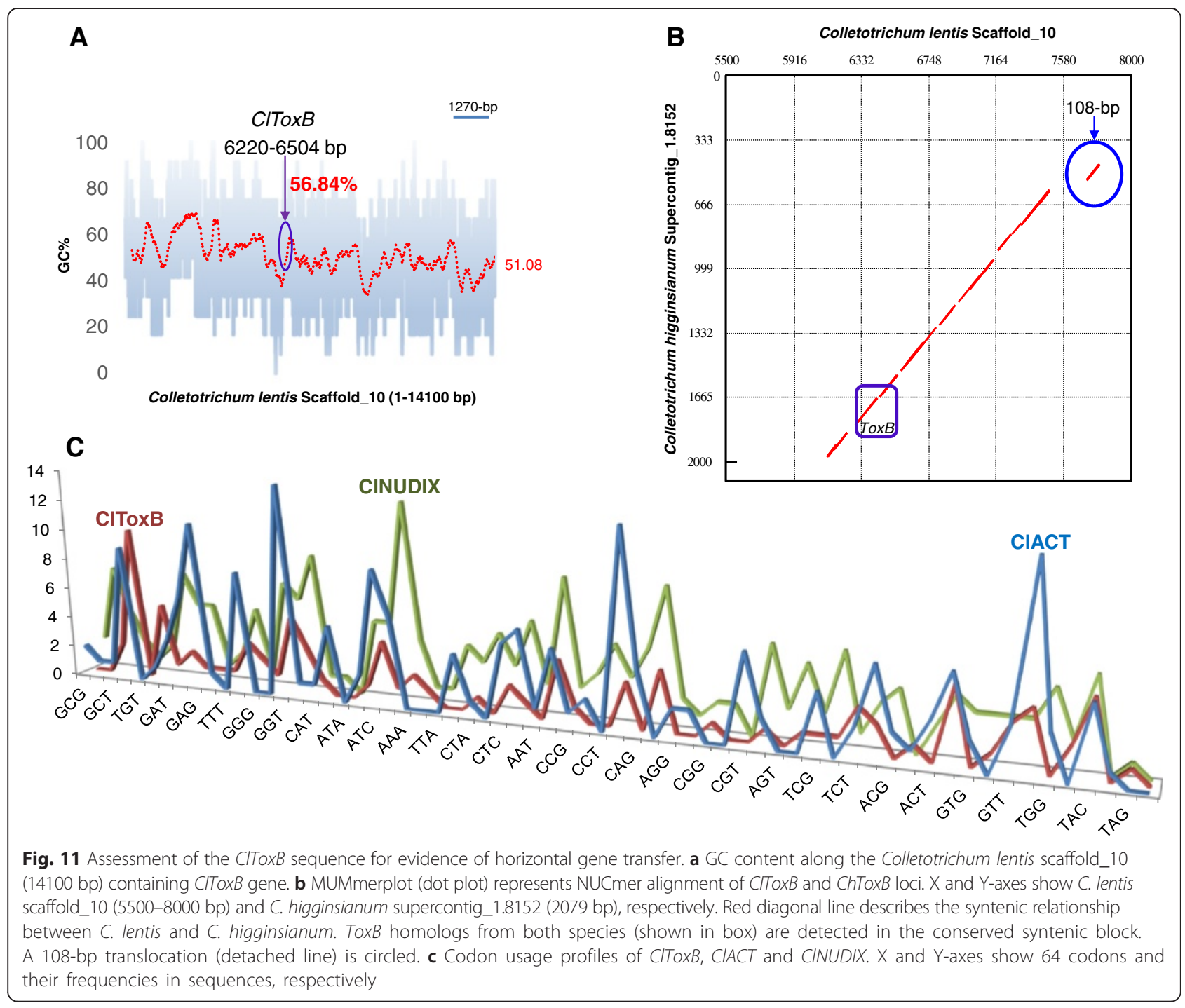

lentis (Figs. 10 and 11). This is different from another, well studied fungal toxin gene, ToxA. This gene transferred from $S$. nodorum to the distantly related $P$. tritici-repentis possibly through conidial anastomosis, and conferred virulence to the latter enabling the fungus to become a serious pathogen on wheat where it causes tan spot disease [20]. This HGT was estimated to have occurred after 1941 because prior to that the tan spot pathogen was not considered a major threat to wheat production.

The transmission of genetic material through HGT is very common in bacteria, but has also been identified in several fungal species, particularly in terms of pathogenicity and virulence genes, resulting in pathogens broadening their host range and/or increasing their virulence. Such changes allow fungi to exploit new ecological niches, but may also result in resistance break-down in agricultural systems. Although the majority of suspected HGT events in fungi have a fungal gene donor, many acquired genes have also been traced back to bacteria, and a very few to plant species [19]. In the genus Colletotrichum, Jaramillo et al. [42] identified eleven HGT events from bacteria including the one encoding argininosuccinate lyase. Some of these HGTs are involved in niche adaptation and virulence. Mining the EST library of $C$. lentis for potential horizontally transferred genes identified the gene Arg of bacterial origin, previously found in several other Colletotrichum spp. [42]. The gene encodes argininosuccinate lyase and is involved in arginine biosynthesis, which is essential for fungal virulence [43]. Considering the high sequence identity with $\mathrm{Arg}$ in several bacterial species, it appears this gene was likely acquired from bacteria by a common ancestor of Colletotrichum species, and may have contributed to an expansion into or colonization of new niches and hosts by increasing virulence in the genus. Although homologs to this gene can be found in other fungal genera (e.g. M. oryzae, data not presented) indicating a 


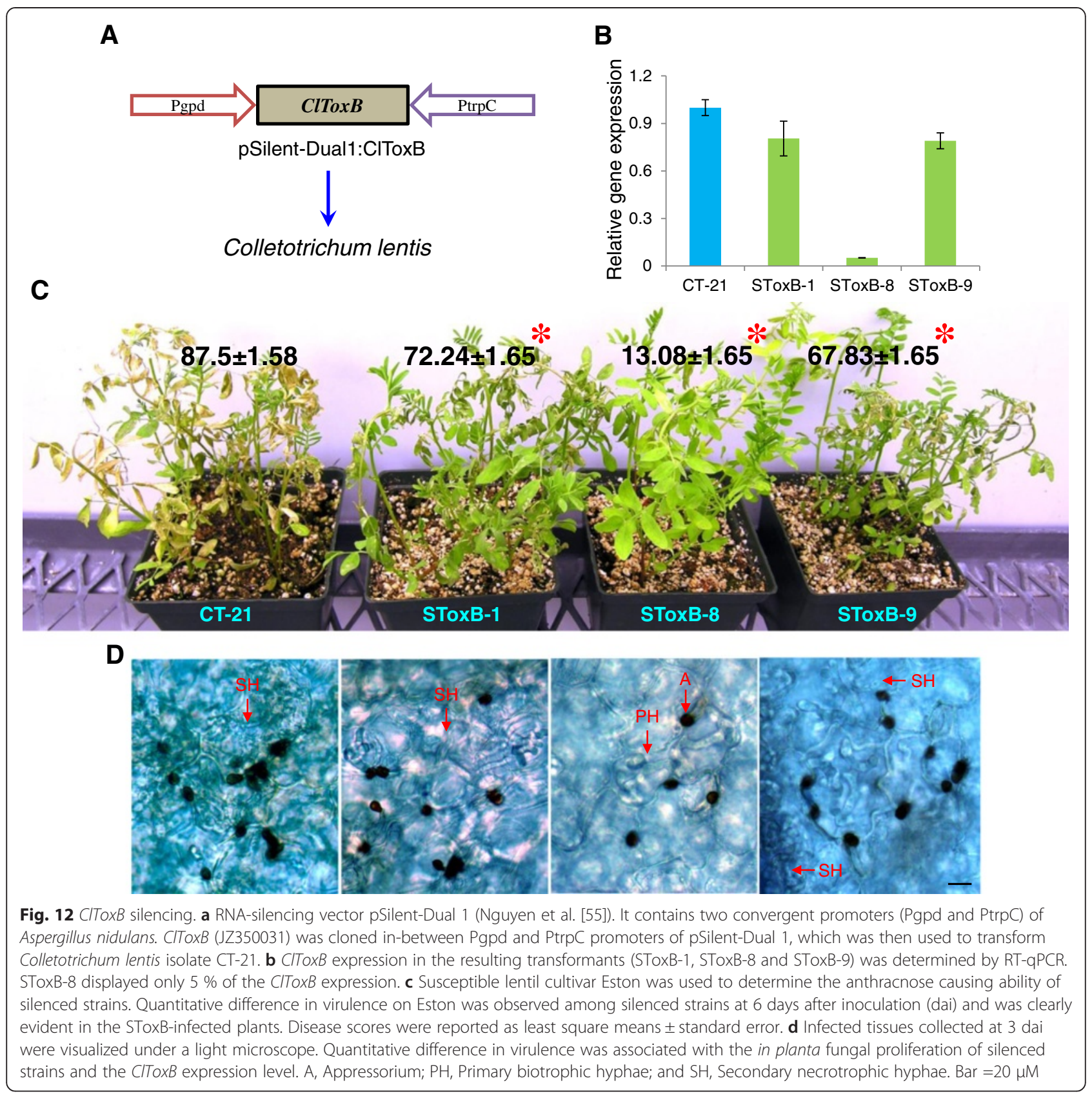

potentially important role in fungi, sequence identity is very low with Arg from Colletotrichum species, suggesting a repeated introduction of this gene into fungal genomes from different bacterial species.

Candidate effectors identified in this study showed no sign of positive selection as no substitution mutation was found $[\omega(\mathrm{dN} / \mathrm{dS})<1]$, which is consistent with the view that partial resistance may not impose enough selection pressure on pathogen populations to allow for diversification and novel races to emerge [44]. The identified SNPs in candidate effectors ClCE6 and ClCE8 were silent, but polymorphisms were used to develop a race differentiation assay (KASPar assays). The ClCE6 KASPar marker could differentiate race 0 isolates from those of race 1 (Fig. 4), suggesting that it may be co-segregating with the virulence governing locus/loci, hence can be used to determine the race identity of $C$. lentis isolates. Race indexing of $C$. lentis isolates is important not only for monitoring the population dynamics of the pathogen, but also for screening germplasm under field conditions. Sources of resistance to race 1 have been identified in the cultivated species $L$. culinaris and have been successfully introgressed into cultivars. High levels of resistance against the more virulent race 0 has only been found in some accessions in the 
Table 5 ClArg homologs

\begin{tabular}{|c|c|c|c|c|c|c|c|c|}
\hline Top 10 hits & Accession & Length (aa) & Putative function & Organism & Query coverage (\%) & Evalue & Taxonomic classification & EC code \\
\hline 1 & ClArg & 488 & Argininosuccinate lyase & Colletotrichum lentis & 99 & - & Fungus & EC 4.3.2.1 \\
\hline 2 & CCF37911 & 488 & Argininosuccinate lyase & Colletotrichum higginsianum & 99 & 0 & Fungus & EC 4.3.2.1 \\
\hline 3 & KDN68815 & 488 & Argininosuccinate lyase & Colletotrichum sublineola & 99 & 0 & Fungus & EC 4.3.2.1 \\
\hline 4 & EFQ25990 & 488 & Argininosuccinate lyase & Colletotrichum graminicola & 99 & 0 & Fungus & EC 4.3.2.1 \\
\hline 5 & XP_007286426 & 487 & Argininosuccinate lyase & Colletotrichum gloeosporioides ${ }^{a}$ & 99 & 0 & Fungus & EC 4.3.2.1 \\
\hline 6 & XP_007595184 & 488 & Argininosuccinate lyase & Colletotrichum fioriniae & 99 & 0 & Fungus & EC 4.3.2.1 \\
\hline 7 & ENH83872 & 487 & Argininosuccinate lyase & Colletotrichum orbiculare & 99 & 0 & Fungus & EC 4.3.2.1 \\
\hline 8 & WP_043836904 & 504 & Argininosuccinate lyase & Roseomonas aerilata & 96 & 0 & Bacterium & EC 4.3.2.1 \\
\hline 9 & WP_003075088 & 522 & Argininosuccinate lyase & Comamonas testosteroni & 96 & 0 & Bacterium & EC 4.3.2.1 \\
\hline 10 & WP_043360792 & 504 & Argininosuccinate lyase & Belnapia sp. F-4-1 & 96 & 0 & Bacterium & EC 4.3.2.1 \\
\hline
\end{tabular}

Top ten hits were obtained by BLASTing peptide sequence of Colletotrichum lentis argininosuccinate lyase against NCBInr protein database

${ }^{\mathrm{a}}$ Two hits were matched to two strains of C. gloeosporiodes. Only one is listed in the table 


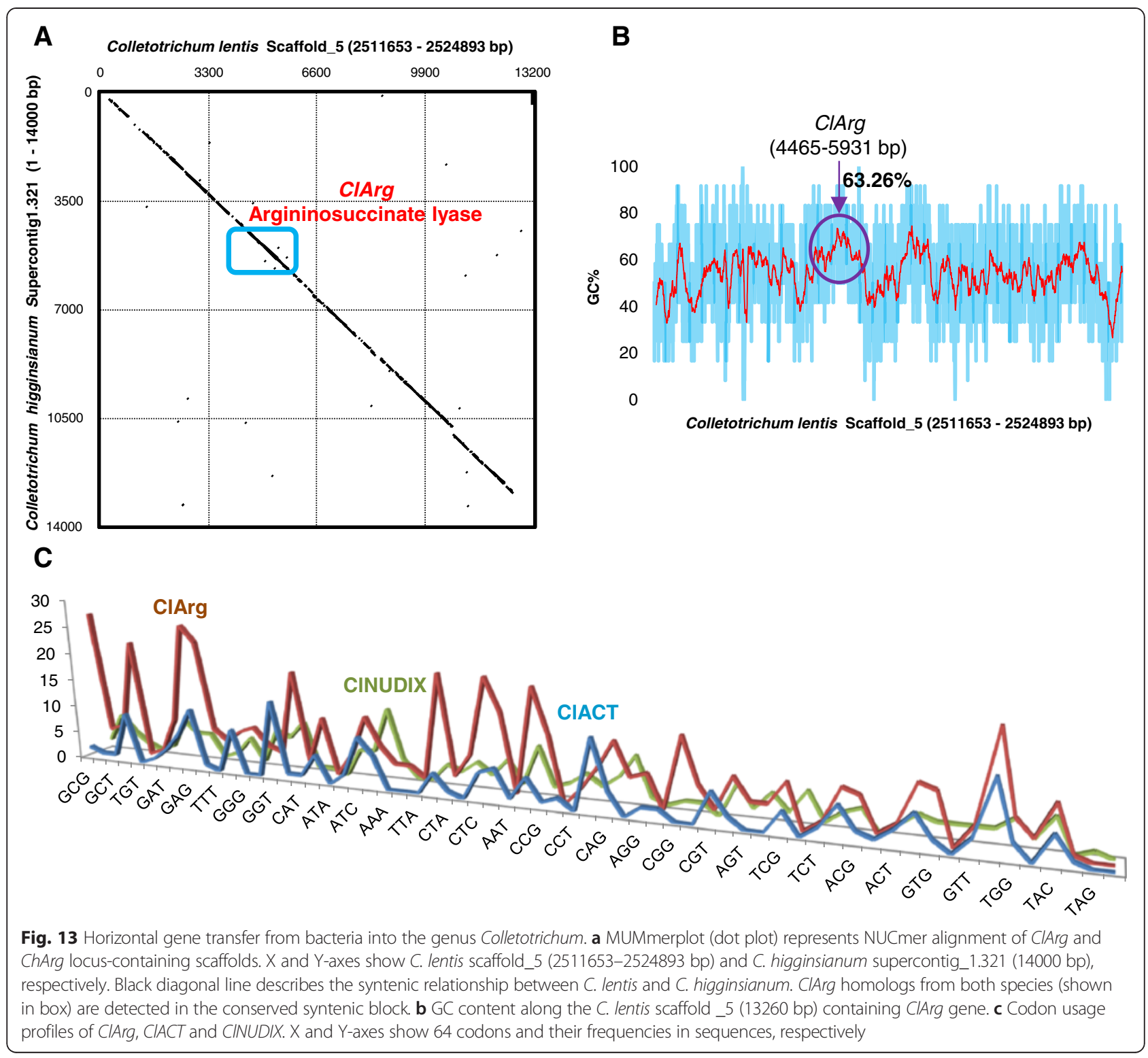

secondary and tertiary gene pools $[45,46]$, and efforts are underway to incorporate race 0 resistance into cultivars.

\section{Conclusions}

EST mining identified a set of in planta expressed candidate effectors. Comparative genomics of effectors revealed no sign of positive selection pressure at the intraspecific level, suggesting that $C$. lentis isolates are under stabilizing selection. Two synonymous SNPs were detected in two of the candidate effectors, one of which (ClCE6) allowed pathogenic race 0 isolates to be differentiated from race 1 isolates. EST mining and comparative genomics also identified the foreign gene Arg encoding argininosuccinate lyase from bacteria among ESTs, which was likely acquired by Colletotrichum from a bacterial species through HGT to improve/enhance virulence. In addition, C. lentis likely secretes a host specific toxin ClToxB in lentil cells during the biotrophy-necrotrophy switch to amplify cell death signals caused the effector CINUDIX and contribute to quantitative differences in virulence between the races 0 and 1 .

\section{Methods}

\section{Bioinformatics}

Previously, we constructed a biotrophy-necrotrophy switch-specific cDNA plasmid library from susceptible lentil cultivar Eston infected with C. lentis isolate CT-21 (race 1) [5]. In this study, 2000 new clones from this library were sequenced to identify candidate effectors. These ESTs were subjected to VecScreen (http://www.ncbi.nlm.nih.gov/ VecScreen) to identify sequences belonging to the vector pBluescript II SK (+). Vector and adapter 
(GAATTCGGCACGGGAGG) sequences were manually trimmed, and the resulting EST sequences were queried against the NCBInr protein database using the BLASTX algorithm (http://www.ncbi.nlm.nih.gov/BLAST) and against the Consortium for the Functional Genomics of Microbial Eukaryotes EST database (http://cogeme. ex.ac.uk) using TBLASTX algorithm [47]. An ORF finder algorithm (http://www.ncbi.nlm.nih.gov/gorf) was employed to predict coding regions of candidate effectors in all six frames $a b$ initio; the longest sequence with a stop codon preceded by an in frame ATG codon was translated into protein sequence. The amino acid sequence was then screened for potential SP and transmembrane helices using SignalP server version 4.1 with default settings (http:// www.cbs.dtu.dk/services/SignalP) and TMHMM server version 2 (http://www.cbs.dtu.dk/services/TMHMM), respectively. Protein sequences containing putative SPs were queried against the NCBI non-redundant protein database using BLASP algorithm (http://www.ncbi.nlm.nih.gov/ BLAST). $N$ - and $O$-linked glycosylation sites were predicted using NetNGlyc 1.0 (http://www.cbs.dtu.dk/services/NetNGlyc ) and NetOGlyc 2.0 servers (http://www.cbs.dtu.dk/ services/NetOGlyc ), respectively. Identified candidate effectors (Table 1) were mapped onto the C. lentis isolate CT-30 (race 0) draft genome (unpublished data) to identify single nucleotide polymorphisms (SNPs) using BioEdit sequence alignment editor [48]. In addition, we re-sequenced the full length cDNA of the previously identified ClToxB of C. lentis-infected lentil (GenBank Accession: JZ350031).

ClCE8 and $\mathrm{ClToxB}$ homologous protein sequences were aligned using Clustal W (Larkin et al. 2007) and ESPrint version 3.0 . MEGA 6 program [49] was used to compute evolutionary distance of ClCE8 homologs. Clustal Waligned ToxB sequences were subjected to Seq2Logo [50] to generate sequence logo.

\section{Plant and fungal materials}

Lentil plants of the Canadian cultivar Eston and compatible $C$. lentis isolate CT-21 were grown and routinely maintained as described previously [5]. CT-21 mycelia (vegetative hyphae) and ungerminated conidia were collected and flash-frozen in liquid nitrogen as described previously [5]. Lentil differential cultivar CDC Robin was used to differentiate isolates belonging to race 1 from race 0 . Race 0 isolates are fully pathogenic on CDC Robin, which shows partial resistance to isolates of race 1 .

\section{Infection time-course}

Leaflets from 3-week-old lentil plants were detached and inoculated in Petri dishes lined with wet filter paper with droplets of CT-21 conidial suspension $\left(5 \times 10^{4}\right.$ conidia $\mathrm{mL}^{-1}$ ). Inoculated leaflets were incubated with $12 \mathrm{~h}$ photoperiod. The progress of fungal infection was microscopically assessed. Infection sites were harvested using a $6 \mathrm{~mm}$ cork borer at 3 time points: Appressorium penetration phase [ $24 \mathrm{~h}$ after infection (hai)], biotrophic stage ( 48 hai, characterized by the presence of fat primary hyphae), and necrotrophic stage (72 hai, characterized by thin secondary hyphae). These leaflet discs were then flash-frozen in liquid nitrogen until required.

\section{Total RNA extraction and RT-qPCR}

Total RNA from mycelia, ungerminated conidia and CT21-infected lentil leaf tissues collected at 24, 48 and 72 hai was isolated using RNeasy Plant Mini kit (Qiagen, Hilden, Germany). After eliminating genomic DNA using RNasefree amplification grade DNase I (Invitrogen, Carlsbad, USA), total RNA $(2 \mu \mathrm{g})$ was reverse transcribed in a $20 \mu \mathrm{L}$ reaction volume using $200 \mathrm{U}$ SuperScript reverse transcriptase (Invitrogen, Carlsbad, USA) following the protocol of the supplier. The resulting cDNA was diluted 10-fold in UltraPure DNase/RNase free-distilled water (Life Technologies, Cergy Pontoise, France).

Real-Time PCR detection platform CFX96 (Bio-Rad, Hercules, USA) was used to quantify the expression of candidate effectors. The actin gene was used as an endogenous control (reference gene). The $5 \mu \mathrm{L}$ reaction contained $2.5 \mu \mathrm{L}$ of $2 \mathrm{X}$ Fast SYBR Green Master mix (Applied Biosystems, Courtaboeuf, France), $200 \mathrm{nmol}$ of each primer and $1 \mu \mathrm{L} \mathrm{cDNA}$ as template. The following thermal conditions were used to quantify the expression: $2 \mathrm{~min}$ of pre-heating at $95^{\circ} \mathrm{C}$ followed by 40 cycles of $10 \mathrm{~s}$ at $95{ }^{\circ} \mathrm{C}$ and $30 \mathrm{~s}$ at $60{ }^{\circ} \mathrm{C}$. For normalization, the threshold cycle $\left(C_{T}\right)$ values of the reference gene were subtracted from the corresponding $\mathrm{C}_{\mathrm{T}}$ values of candidate effectors, generating $\Delta \mathrm{C}_{\mathrm{T}}$ values. The relative expression of candidate effectors was calculated by the comparative $\mathrm{C}_{\mathrm{T}}$ method [51] using a $\Delta \mathrm{C}_{\mathrm{T}}$ value obtained for vegetative hyphae as a calibrator. All relative expression values of genes were reported as means \pm standard errors of the means on a 2-log scale (Additional file 1). Average fold change values were summarized as a clustergram. Primers used in RT-qPCR analyses are listed in Additional file 2.

\section{SNP genotyping}

Genomic DNA was extracted from mycelia of 52 C. lentis isolates (Table 2) using DNeasy Plant Mini Kit (Qiagen, Hilden, Germany). Two forward primers in which the 3-prime end corresponds to one of the SNP alleles (Allele $\mathrm{C}$ corresponds to race $0, \mathrm{~T}$ to race 1 ) and the 5 prime end is tailed with fluorophore FAM ( $\mathrm{C}$ allelespecific primer) and VIC ( $\mathrm{T}$ allele-specific primer), and a common reverse primer were used for each candidate effector, ClCE6 and ClCE8 (Additional file 3). KASPar SNP genotyping was performed on a CFX384 Real-Time System (Bio-Rad, Hercules, USA) following the protocol 
of the manufacturer (KBioscience, Hoddeston, UK). Percentage endpoint relative fluorescence was used to generate an allele discrimination plot. Race identity ( 0 or 1$)$ of all 52 isolates based on SNP genotyping are listed in the Table 2.

\section{Phenotyping of $C$. lentis isolates}

Lentil differential cultivar CDC Robin was inoculated in pathogenicity assays with various $C$. lentis isolates collected from Saskatchewan and Manitoba, Canada, to determine their race identity (Table 2). Lentil cultivar Eston susceptible to both races was used as a susceptible control. Phenotyping was conducted as described previously [7].

\section{CIToxB in planta expression}

The plasmid pGR106 used in the study was kindly provided by Dr. David Baulcombe (University of Cambridge, Cambridge, UK). Primers used to construct pGR106$\mathrm{ClToxB}$ and pGR106-ClToxB $\triangle \mathrm{SP}$ vectors are listed in Additional file 4 . The ClToxB open reading frame with and without SP (ClToxB $\triangle \mathrm{SP})$ were cloned into pCR2.1 (Invitrogen, Carlsbad, USA) and sequenced. Confirmed DNA sequences were digested with the restriction enzymes ClaI and NotI and ligated into the potato virus Xbased binary vector pGR106. Binary constructs were then used to transform Agrobacterium tumefaciens strain GV3101 carrying the helper plasmid pJIC Sa_Rep (pSoup). Infiltration assays with recombinant $A$. tumefaciens were performed on 4-6 weeks old tobacco (Nicotiana tabacum) as described previously [5]. The macroscopic phenotype was monitored from 2 to $10 \mathrm{dpi}$. Photographs were taken from 2 to $10 \mathrm{dpi}$.

\section{Phylogeny and comparative genomics}

We retrieved ITS-5.8S, GAPDH, CHS-1, HIS3, ACT, TUB2 and ToxB sequences from the NCBI GenBank database for $C$. lentis, $C$. higginsianum, $C$. orbiculare, $C$. gloeosporiodes, C. fioriniae, Magnaporthe oryzae and $P$. tritici-repentis. Phylogenetic analysis was conducted with the Bayesian inference (BI) method using MrBayes 3.1 [52]. The GTR + G + I nucleotide substitution model was used to infer the species tree based on the loci listed above and ToxB tree based on ToxB homologs. This analysis ran for 1 million generations using four Markov chain Monte Carlo chains ( 3 hot and 1 cold) and trees were sampled at every 100 generations. Twenty-five percent topologies were burned out to construct the consensus species and ToxB consensus trees. Neighbor Joining (NJ), Maximum Parsimony (MP) and Maximum Likelihood (ML) trees were constructed using MEGA6 [49].

ClToxB and ChToxB, and ClArg and ChArg loci containing scaffolds were retrieved from the draft genomes of C. lentis and C. higginsianum [3]. Comparative genomics was performed using MUMmer software package 3 [53]. The NUCmer function of MUMmer package was used to align scaffolds, and the MUMmerplot function was used to visualize the alignment as a dot plot.

We explored the possibility of HGT of ToxB in $C$. lentis using 4 criteria: Phylogenetic distribution, GC content of the pseudomolecule (chromosome), syntenic relationship between ToxB loci and codon usage [54]. A $\mathrm{BI}$ algorithm was used to generate a species tree from multiple loci, including ITS-5.8S, GAPDH, CHS-1, HIS3, ACT and TUB2, and a ToxB-based gene tree obtained from $C$. lentis, $C$. higginsianum, $C$. orbiculare, $C$. gloeosporiodes, C. fioriniae, Magnaporthe oryzae and $P$. tritici-repentis. Other algorithms, such as NJ, MP and ML resulted in nearly similar topologies of both, species and ToxB gene phylogenetic trees.

\section{CIToxB silencing and pathogenicity testing}

The plasmid pSilent-Dual 1 [55] used in this study was kindly provided by Dr. Hitoshi Nakayashiki (Kobe University, Kobe, Japan). The ClToxB open reading frame was amplified using the primer ClToxBSF/ ClToxBSR and cloned into the EcoRV site of the pSilent-Dual 1. Primers used in the study are listed in the Additional file 5. The plasmid contains two convergent RNA polymerase II promoters, Pgpd and PtrpC, from Aspergillus nidulans, thereby producing sense and antisense RNA pools of ClToxB. pSilent-Dual 1-ClToxB was used to transform $C$. lentis spheroplasts as described previously [27]. Resulting geneticin-resistant transformants were selected and subjected to RT-qPCR to quantify $C l T o x B$ expression level as described above. Three silenced strains were used for pathogenicity testing.

Twenty-one-day-old Eston plants were sprayed with $5 \times 10^{4}$ conidia per $\mathrm{mL}(3 \mathrm{~mL}$ per plant) and anthracnose symptoms on leaves and stems were scored on a 0 to 10 scale (with $10 \%$ increments) where 0 indicated no lesion and 10 was equivalent to $91-100 \%$ disease severity. Isolates (CT-21 and silenced strains) were assigned to experimental units in an RCBD with 3 independent biological replications each consisting of 4 plants in one pot. Disease severity scores were collected at 6 dai from each plant of the three biological replications, values were transformed into \% values using mid-class values and subjected to analysis of variance using the mixed model procedure (PROC MIXED) of SAS v.9.3 (SAS Institute, Cary, USA) to determine whether there were significant differences in disease severity scores between the strain with knock-down ClToxB mRNAs and the wild-type. Disease severity scores were reported as least squares means of \% values \pm standard error. The difference between treatments was determined by Fisher's least significant difference (LSD, $\mathrm{p} \leq 0.05$ ). 


\section{Availability of supporting data}

All the supporting data are included as additional files with the online version of this article.

\section{Additional files}

\section{Additional file 1: RT-qPCR expression profiling of candidate}

effectors of Colletotrichum lentis. Average $C_{T}, \Delta C_{T}$, and $2^{\wedge}\left(-\Delta C_{T}\right)$ fold change and fold regulation values are listed in separate sheets. (XLS $37 \mathrm{~kb}$ )

Additional file 2: RT-qPCR primers used to quantify expression of Colletotrichum lentis candidate effectors in an infection time-course on lentil cultivar Eston. (XLSX $10 \mathrm{~kb}$ )

Additional file 3: KASPar markers (CICE6 and CICE8). Two forward primers in which the 3-prime end corresponds to one of the SNP alleles (Allele $\mathrm{C}$ corresponds to race 0 and $\mathrm{T}$ to race 1 ) and the 5 -prime end is tailed with fluorophore FAM (C allele) and VIC (T allele), and a common reverse primer were used for each candidate effector. (XLSX $9 \mathrm{~kb}$ )

\section{Additional file 4: Primers used in transient expression vector} construction. Primer set CT30ToxBF/R was used to clone CIToxB into the binary vector pGR106 whereas CIToxB without signal peptide was cloned into pGR106 using the primer set CT30ToxB $\triangle S P / C T 30 T o x B R$. (XLSX $9 \mathrm{~kb}$ )

Additional file 5: Primers used to construct pSilent-Dual 1-CIToxB construct. Underline sites are EcoRV restriction sites and used to clone CIToxB ORF into the plasmid pSilent-Dual 1. (XLSX $9 \mathrm{~kb})$

\section{Competing interests}

The authors declare that they have no competing interests.

\section{Authors' contribution}

$\mathrm{SB}, \mathrm{VB}$ and $\mathrm{CP}$ coordinated the project and wrote the manuscript. VB performed the experiments. RM and VB analyzed the data. All authors read and approved the final manuscript.

\section{Acknowledgments}

Technical support for this study was provided by Darlene Klassen. This work was supported by the National Science and Engineering Research Council of Canada and by the Saskatchewan Pulse Growers through an NSERC-CRD grant. We thank Dr. David Baulcombe for providing plasmid pGR106 and Agrobacterium tumefaciens strain GV3101, Dr. Sophien Kamoun for pGR106-INF1 construct and Dr. Hitoshi Nakayashiki for plasmid pSilent-Dual 1.

Received: 1 May 2015 Accepted: 7 August 2015

Published online: 22 August 2015

\section{References}

1. Sutton BC. The coelomycetes: Fungi imperfecti with pycnidia, acervuli and stromata. Kew: Commonwealth Mycological Institute; 1980

2. Damm U, Woudenberg JHC, Cannon PF, Crous P. Colletotrichum species with curved conidia from herbaceous hosts. Fungal Div. 2009;39:45-87.

3. O'Connell RJ, Thon MR, Hacquard S, Amyotte SG, Kleemann J, Torres MF, et al. Lifestyle transitions in plant pathogenic Colletotrichum fungi deciphered by genome and transcriptome analyses. Nat Genet. 2012;44:1060-5.

4. Damm U, O'Connell RJ, Groenewald JZ, Crous P. The Colletotrichum destructivum species complex - hemibiotrophic pathogens of forage and field crops. Studies Mycol. 2014;79:49-84.

5. Bhadauria V, Banniza S, Vandenberg A, Selvaraj G, Wei Y. EST mining identifies proteins putatively secreted by the anthracnose pathogen Colletotrichum truncatum. BMC Genomics. 2011;12, e327.

6. Buchwaldt L, Anderson KL, Morrall RAA, Gossen BD, Bernier CC. Identification of lentil germplasm resistant to Colletotrichum truncatum and characterization of two pathogen races. Phytopathology. 2004:94:236-43.

7. Armstrong-Cho C, Wang J, Wei Y, Banniza S. The infection process of two pathogenic races of Colletotrichum truncatum on lentil. Can J Plant Pathol. 2012;34(1):58-67.
8. Huitema E, Bos JIB, Tian M, Win J, Waugh ME, Kamoun S. Linking sequence to phenotype in Phytophthora-plant interactions. Trends Microbiol. 2004;12:193-200

9. Kamoun S. A catalogue of the effector secretome of plant pathogenic oomycetes. Annu Rev Phytopathol. 2006;44:41-60.

10. Kamoun S. Groovy times: Filamentous pathogen effectors revealed. Curr Opin Plant Biol. 2007;10:358-65.

11. Whisson SC, Boevink PC, Moleleki L, Avrova AO, Morales JG, Gilroy EM. A translocation signal for delivery of oomycete effector proteins into host plant cells. Nature. 2007:450:115-8.

12. Kale SD, Gu B, Capelluto DGS, Dou D, Feldman E, Rumore A. External lipid PI3P mediates entry of eukaryotic pathogen effectors into plant and animal host cells. Cell. 2010;142:284-95.

13. Chisholm ST, Coaker G, Day B, Staskawicz BJ. Host-microbe interactions: Shaping the evolution of the plant immune response. Cell. 2006;124:803-14.

14. Jones JDG. Dangl JL (2006). The plant immune system. Nature. 2006;444:323-9.

15. Lamari L, Strelkov SE, Yahyaoui A, Orabi J, Smith RB. The identification of two new races of Pyrenophora tritici-repentis from the host centre of diversity confirms a one-to-one relationship in tan spot of wheat. Phytopathology. 2003;93:391-6

16. Lamari L, Strelkov SE. The wheat/Pyrenophora tritici-repentis interaction: progress towards an understanding of tan spot disease. Can J Plant Pathol. 2010;32:4-10.

17. Kidwell MG. Lateral transfer in natural populations of eukaryotes. Annu Rev Genet. 1993:27:235-56.

18. Lawrence JG, Roth JR. Selfish operons: horizontal transfer may drive the evolution of gene clusters. Genetics. 1996;143:1843-60.

19. Soanes D, Richards TA. Horizontal gene transfer in eukaryotic plant pathogens. Annu Rev Phytopathol. 2014;52:583-614.

20. Friesen TL, Stukenbrock EH, Liu ZH, Meinhardt SW, Ling H, Faris JD, et al. Emergence of a new disease as a result of interspecific virulence gene transfer. Nat Genet. 2006:38:953-6.

21. Perfect SE, O'Connell R, Green EF, Doering-Saad C, Green JR. Expression cloning of a fungal proline-rich glycoprotein specific to the biotrophic interface formed in the Colletotrichum -bean interaction. Plant J. 1998;15:273-9.

22. Bolton MD, van Esse HP, Vossen JH, De Jonge R, Stergiopoulos I, Stulemeijer IJE, et al. The novel Cladosporium fulvum lysin motif effector Ecp6 is a virulence factor with orthologues in other fungal species. Mol Microbiol. 2008;69:119-36.

23. Stephenson SA, Hatfield J, Rusu AG, Maclean DJ, Manners JM. CgDN3 an essential pathogenicity gene of Colletotrichum gloeosporioides necessary to avert a hypersensitive-like response in the host Stylosanthes guianensis. Mol Plant Microbe In. 2000;13:929-41.

24. Krijger JJ, Horbach R, Behr M, Schweizer P, Deising HB, Wirsel SG. The yeast signal sequence trap identifies secreted proteins of the hemibiotrophic corn pathogen Colletotrichum graminicola. Mol Plant Microbe In. 2008:21(10):1325-36.

25. Kleemann J, Takahara H, Stüber K, O'Connell R. Identification of soluble secreted proteins from appressoria of Colletotrichum higginsianum by analysis of expressed sequence tags. Microbiology. 2008;154:1204-17.

26. Takahara H, Dolf A, Endl E, O'Connell R. Flow cytometric purification of Colletotrichum higginsianum biotrophic hyphae from Arabidopsis leaves for stage-specific transcriptome analysis. Plant J. 2009;59:672-83.

27. Bhadauria V, Banniza S, Vandenberg A, Selvaraj G, Wei Y. Overexpression of a novel biotrophy-specific Colletotrichum truncatum effector CtNUDIX in hemibiotrophic fungal phytopathogens causes incompatibility with their host plants. Eukaryot Cell. 2013;12(1):2-11.

28. Kleemann J, Rincon-Rivera $\amalg$, Takahara H, Neumann U, van Themaat EVL, van der Does HC. Sequential delivery of host-induced virulence effectors by appressoria and intracellular hyphae of the phytopathogen Colletotrichum higginsianum. PLoS Pathog. 2012;8(4), e1002643.

29. Wang C, St. Leger RJ. A collagenous protective coat enables Metarhizium anisopliae to evade insect immune responses. Proc Natl Acad Sci U S A. 2006;103(17):6647-52.

30. Chatterjee S, Mayor S. The GPI-anchor and protein sorting. Cell Mol Life Sci. 2001:58:1969-87.

31. Litvintseva AP, Henson JM. Cloning, characterization, and transcription of three laccase genes from Gaeumannomyces graminis var. tritici, the take-all fungus. Appl Environ Microbiol. 2002;68(3):1305-11.

32. Stergiopoulos I, de Wit PJGM. Fungal effector proteins. Annu Rev Phytopathol. 2009;47:233-63. 
33. Krzywinski MI, Schein JE, Birol I, Connors J, Gascoyne R, Horsman D, et al Circos: an information aesthetic for comparative genomics. Genome Res. 2009;19:1639-45

34. Liu T, Chen G, Min H, Fu-cheng Lin. MoFLP1, encoding a novel fungal fasciclin-like protein, is involved in conidiation and pathogenicity in Magnaporthe oryzae. J Zhejiang Univ Sci B. 2009;10(6):434-44.

35. Kamoun $S$, van West $P$, de Jong AJ, de Groot KE, Vleeshouwers VG, Govers F. A gene encoding a protein elicitor of Phytophthora infestans is down-regulated during infection of potato. Mol Plant Microbe In. 1997;10(1):13-20.

36. Dawkins R. The Extended Phenotype: The Long Reach of the Gene. 1st ed. Oxford: Oxford University Press; 1999.

37. Ellis JG, Rafiqi M, Gan P, Chakrabarti A, Dodds PN. Recent progress in discovery and functional analysis of effector proteins of fungal and oomycete plant pathogens. Curr Opin Plant Biol. 2009;12:399-405.

38. Vleeshouwers VGAA, Raffaele $\mathrm{S}$, Vossen JH, Champouret N, Oliva R, Segretin ME, et al. Understanding and exploiting late blight resistance in the age of effectors. Annu Rev Phytopathol. 2011:49:507-31.

39. Vleeshouwers VGAA, Oliver RP. Effectors as tools in disease resistance breeding against biotrophic, hemibiotrophic and necrotrophic pathogens. Mol Plant Microbe In. 2014;27(3):196-206.

40. Elkins T, Hortsch M, Bieber AJ, Snow PM, Goodman CS. Drosophila fasciclin I is a novel hemophilic adhesion molecule that along with fasciclin III can mediate cell sorting. J Cell Biol. 1990;110(5):1825-32.

41. Choi GH, Larson TG, Nuss DL. Molecular analysis of the laccase gene from the chestnut blight fungus and selective suppression of its expression in an isogenic hypovirulent strain. Mol Plant Microbe In. 1992:5:119-28.

42. Jaramillo VDA, Sukno SA, Thon MR. Identification of horizontally transferred genes in the genus Colletotrichum reveals a steady tempo of bacterial to fungal gene transfer. BMC Genomics. 2014;16:e2.

43. Namiki F, Matsunaga M, Okuda M, Inoue I, Nishi K, Fujita $Y$, et al. Mutation of an arginine biosynthesis gene causes reduced pathogenicity in Fusarium oxysporum f. sp. melonis. Mol Plant Microbe In. 2001;14:580-4

44. James RV, Fry WE. Potential for Phytophthora infestans populations to adapt to potato cultivars with rate-reducing resistance. Phytopathology. 1983;73:984-8

45. Tullu A, Buchwaldt L, Lulsdorf M, Banniza S, Barlow B, Slinkard AE, et al. Sources of resistance to anthracnose (Colletotrichum truncatum) in wild Lens species. Genet Resour Crop Ev. 2006;53:111-9.

46. Shaikh R, Diederichsen A, Harrington M, Adam J, Conner RL, Buchwaldt L. New sources of resistance to Colletotrichum truncatum race $\mathrm{Ct0}$ and $\mathrm{Ct} 1$ in Lens culinaris Medikus subsp. culinaris obtained by single plant selection in germplasm accessions. Genet Resour Crop Ev. 2013;60(1):193-201.

47. Altschul SF, Warren G, Webb M, Myers EW, Lipman DJ. Basic local alignment search tool. J Mol Biol. 1990;215:403-10.

48. Hall TA. BioEdit: a user-friendly biological sequence alignment editor and analysis program for Windows 95/98/NT. Nucleic Acids Symp Ser. 1999;41:95-8.

49. Tamura K, Stecher G, Peterson D, Filipski A, Kumar S. MEGA6: Molecular Evolutionary Genetics Analysis Version 6.0. Mol Biol Ev. 2013;30:2725-9.

50. Thomsen MCF, Nielsen M. Seq2Logo: a method for construction and visualization of amino acid binding motifs and sequence profiles including sequence weighting, pseudo counts and two-sided representation of amino acid enrichment and depletion. Nucleic Acids Res. 2012;40(W1):W281-7

51. Livak KJ, Schmittgen TD. Analysis of relative gene expression data using real-time quantitative PCR and the $2_{T}^{-\Delta \Delta C}$ method. Methods. 2001;25:402-8.

52. Huelsenbeck JP, Ronquist F. MRBAYES: bayesian inference of phylogenetic trees. Bioinformatics. 2001;17(8):754-5.

53. Kurtz S, Phillippy A, Delcher AL, Smoot M, Shumway M, Antonescu C. Versatile and open software for comparing large genomes. Genome Biol. 2004;5(2):R12.

54. Fitzpatrick DA. Horizontal gene transfer in fungi. FEMS Microbiol Lett. 2012;329(1):1-8.

55. Nguyen QB, Kadotani N, Kasahara S, Tosa Y, Mayama S, Nakayashiki H. Systematic functional analysis of calcium-signalling proteins in the genome of the rice-blast fungus, Magnaporthe oryzae, using a highthroughput RNA-silencing system. Mol Microbiol. 2008;68(6):1348-65.

\section{Submit your next manuscript to BioMed Central and take full advantage of:}

- Convenient online submission

- Thorough peer review

- No space constraints or color figure charges

- Immediate publication on acceptance

- Inclusion in PubMed, CAS, Scopus and Google Scholar

- Research which is freely available for redistribution 